\title{
An Image Based Model of Fluid Flow Through Lymph Nodes
}

Laura J. Cooper ${ }^{1,3}$, James Heppell ${ }^{1}$, Geraldine F. Clough ${ }^{2,3}$, Bharathram Ganapathisubramani ${ }^{1,3}$, Tiina Roose ${ }^{1,3}$

${ }^{1}$ Faculty of Engineering and the Environment, University of Southampton, UK

${ }^{2}$ Faculty of Medicine, University of Southampton, UK

${ }^{3}$ Institute for Life Sciences, University of Southampton, UK

Corresponding Author:

L. J. Cooper

Faculty of Engineering and Environment, University of Southampton, Highfield Campus, Southampton, SO17 1BJ, UK

Tel.: +44(0)2380 597665

Fax: +44(0) 2380593016

laura.cooper@soton.ac.uk

G. F. Clough

Faculty of Medicine, University of Southampton, Southampton General Hospital, Southampton, SO16 6YD, UK

J. Heppell, B. Ganapathisubramani and T. Roose

Faculty of Engineering and the Environment, University of Southampton, Highfield Campus, Southampton, SO17 1BJ, UK

\section{Abstract}

The lymphatic system returns fluid to the blood stream from the tissues to maintain tissue fluid homeostasis. Lymph nodes distributed throughout the system filter the lymphatic fluid. The afferent and efferent lymph flow conditions of lymph nodes can be measured in experiments, however it is difficult to measure the flow within the nodes. In this paper we present an image based modelling approach to investigate how the internal structure of the node affects the fluid flow pathways within the node. Selective plane illumination microscopy images of murine lymph nodes are used to identify the geometry and structure of the tissue within the node and to determine the permeability of the lymph node interstitium to lymphatic fluid. Experimental data are used to determine boundary conditions and optimise the parameters for the model. The numerical simulations conducted within the model are implemented in COMSOL Multiphysics, a commercial finite element analysis software. The parameter fitting resulted in the estimate that the average permeability for lymph node tissue is of the order of magnitude of $10^{-11} \mathrm{~m}^{2}$. Our modelling shows that the flow predominantly takes a direct path between the afferent and efferent lymphatics and that fluid is both filtered and absorbed across the blood vessel boundaries. The amount that is absorbed or extravasated in the model is dependent on the efferent lymphatic lumen fluid pressure.

\section{Keywords:}

Lymph nodes, image based modelling, porous media, finite element modelling 
46 We gratefully acknowledge the assistance of Jürgen Mayer (EMBL/CRG Systems Biology

47 Research Unit, Center for Genomic Regulation (CRG) and UPF, Dr. Aiguader 88, 08003,

48 Barcelona, Spain) who provided the lymph node images. LJC was funded partially by a PhD

49 studentship awarded by the Institute of Life Sciences and supplemented with a studentship

50 awarded by the Faculty of Engineering and the Environment at the University of Southampton.

51 The authors acknowledge the use of the IRIDIS High Performance Computing Facility and the

$52 \mu$-VIS centre for computed tomography, at the University of Southampton in the completion of

53 this work.

54 
Lymph nodes are an important part of the immune system. They filter lymphatic fluid, are a site for transfer of immune cells from the blood to the lymphatic fluid, and regulate the protein content of the fluid. The implied pathways for fluid flow through the lymph node have been inferred from its structure and by tracking particulate matter through the node (Ohtani et al., 2003, Gretz et al., 2000). In this paper we present an image based model to characterise the fluid flow through a lymph node.

The structure of lymph nodes is complex. Lymph nodes consist of spaces (lymphatic labyrinths) lined with lymphatic endothelial cells within a porous matrix that allows fluid circulation. The majority of the node parenchyma is made up of lymphocytes and reticular cells within a matrix of collagen fibers. The morphology of the reticular cell network is regulated by fluid flow and the flow also plays a role in directing the migration of cells such as mature dendritic cells and naive T cells (Tomei et al., 2009). The fluid flow through the node has been linked to reducing the preference of cells to migrate within the node, causing more of the immune cells to exit the node (Grigorova et al., 2010). As the lymph nodes are involved in antigen sensing and immune cell activation, understanding how fluid is transported through the node could be useful for developing knowledge of how fluid movement affects the immune response of the node. Adjacent to the lymphatic labyrinths are high endothelial venules (HEVs), through which circulating lymphocytes enter the node parenchyma. The whole node is surrounded by a fibrous capsule under which lies the subcapsular space.

Fluid flow through the parenchyma determines the interstitial protein concentration and hence the transport of fluid across the blood vessel wall. Lymphatic fluid entering the node often has a lower protein concentration than the lymph leaving the node (Adair et al., 1982). Soluble molecules have been injected into rat and mouse lymph nodes (Gretz et al., 2000) and the particles tracked as they progressed through the node. In mice, Gretz et al. (2000) found that the size of the particles determined what path they took through the node; larger particles (2000 kD) were restricted to the subcapsular and medullary sinuses, whereas smaller particles (10 kD) were able to enter the reticular fibre network. Since a role of the lymph node is to filter the lymphatic fluid, tracing particles through the node does not necessary trace the fluid pathways. In this paper we use computational modelling to investigate how the structure of the node effects the direction of fluid transport within the lymph node.

Previous lymph node computational models have focused on the organisation and transport of immune cells through the node. Beltman et al. (2007) created a time dependent model to investigate $\mathrm{T}$ cell motility to describe how they interact with dendritic cells, which present antigens to $T$ cells. The simulation domain was assumed to be a tightly packed cuboid environment containing the reticular network, dendritic cells and T cells. Bogle and Dunbar $(2008,2010,2012)$ have developed a model of T cell movement in a spherical lymph node domain over a 6 hour period. 
In this paper we present a model of fluid transport though a whole lymph node based on images of two murine lymph nodes obtained using selective plane illumination microscopy (SPIM) (Mayer 2012). An advantage of this method is that the domain is not assumed to be an idealised shape but is based on experimental images. The parameters required for the model are lymph node tissue permeability, hydraulic conductivity of the blood vessel walls within the node, blood pressure and inlet and outlet lymphatic flow conditions. Where possible, these values have been obtained from the literature, and where these values cannot be found they have been approximated from similar materials or optimised to the experimental data available.

\subsection{Data used in this study}

In the 1980's experiments were carried out on lymph nodes to find out how the composition of the lymph changed as it passed through the node. Adair et al., (1982), Adair and Guyton (1983, 1985 ) isolated popliteal lymph nodes from dogs and cannulised an efferent and an afferent lymphatic to assess the flow; other afferent and efferent lymphatic vessels were ligated. The lymph nodes were then perfused at physiological flow rates through the afferent lymphatic. The efferent lymphatic pressure was maintained at a hydrostatic pressure $0 \mathrm{mmHg}$, considering atmospheric pressure as gauge pressure. A branch of the lateral saphenous vein was cannulised for the measurement and control of venous pressure.

Adair and Guyton (1985) varied the efferent lymphatic pressure to see how this effected the protein concentration of the efferent lymph fluid. The authors also recorded the changes in the efferent fluid flow rate in response to changes in fluid pressure. These results were used as inputs for our lymph node model.

For the first four nodes in the experiments presented in Adair and Guyton (1985), the efferent lymphatic pressure was increased in 6 steps from 0 to approximately $2170 \mathrm{~Pa}$. We calculated the mean values for the afferent lymphatic pressure, efferent lymphatic pressure, afferent lymphatic flow rate, efferent lymphatic flow rate and venous pressure. The mean efferent lymphatic pressure, afferent lymphatic flow rate and venous pressure were used as inputs in our model. We used the afferent lymphatic pressure and efferent lymphatic flow rate to estimate the unknown parameter values that represented the permeability of the parenchyma, the hydraulic conductivity of the blood vessel walls and the colloid osmotic pressure difference across the blood vessel walls.

\section{Methodology}

\subsection{Image Processing}

The images used in our study were obtained from Jürgen Mayer who imaged mouse popliteal lymph nodes using selective plane illumination microscopy (SPIM); for more information about the experimental protocol see Mayer et al. (2012). In this work, two nodes, one from a wild type mouse (WT) and one from a plt/plt mouse (PLT) (a mutant mouse that lacks certain proteins in the T-cell zone resulting in a decreased accumulation of dendritic cells in this zone) were imaged. 
161

162

163

164

165

166

167

168

169

170

171

172

173

174

175

Alexa594-coupled MECA-79 mAB was injected into the mouse to visualise the high endothelial venules (HEVs) (Mayer et al., 2012). The images of the lymph nodes had a voxel size of $1.29 \mathrm{x}$ $1.29 \times 5 \mu \mathrm{m}$ and were received in 16 bit grayscale tiff format.

To create a computational mesh for finite element modelling of fluid flow through a lymph node, the raw image data had to be segmented and smoothed. This was achieved in the following manner: The images were processed to enable segmentation by Avizo Fire ${ }^{1}$ and analysed as follows. The first stage of image processing was carried out using Fiji ${ }^{2}$ (Schindelin et al, 2012) to convert the image stack to 8 bit greyscale. This significantly reduced the processing time and necessary computational resources. A mean filter with a 4 pixel radius was applied to remove noise from the images. The brightness and contrast adjustment tool in Fiji was used in automatic mode so that the features of the node were visually distinguishable; in particular the HEVs appeared as bright white. The results of these processes are compared to the original image, Figure 1a, in Figure 1b. The end slices were removed from the stack so that the final stack only contained images of the node without any surrounding material. Semi-manual segmentation of the geometry was carried out in Avizo Fire using the magnetic lasso tool. The outline of the node was segmented every 20 slices, except where there were features that required more frequent selections. The selections were then interpolated so that the entire node was selected. Views from other planes were checked to confirm the selection. This segmentation was then used to create a mask that formed the outline of the node, see Figure 1c. The mask stack and the filtered image stack were imported into Fiji. Two new image stacks were created; one of the node on a black background, by subtracting the inverted mask from the image stack; and one of the node on a white background, by adding the inverted mask to the image stack. The node on the white background can be seen in Figure 1d.

The image stack of the node with the black background was imported into Fiji. By selecting a line that crossed a single HEV, the grey scale profile along the line was plotted, see Figures $2 f$ and $2 \mathrm{~g}$. From this, the approximate threshold was found to segment out the HEVs (140 in this case). The brightness and contrast of the image stack were adjusted so that everything above 140 was white and everything below was black. This resulted in another stack of images, which only showed the HEVs. The fill holes tool was applied and then the "remove outliers" tool for radius of 4 pixels. The erosion tool was applied 4 times and after the dilate tool 4 times. This simplified the HEVs structure and removed small features that would make computational mesh generation difficult. This resulted in a mask of white HEVs on a black background. In order for the meshing software to create a boundary between the lymph node tissue and the HEVs, thresholding would be used. In order that the HEVs had a different value from the white node mask the HEV value was reduced by dividing it by an arbitrary number (in this case 4). The mask of the HEVs was subtracted from the mask of the node, see Figure $1 \mathrm{e}$.

\footnotetext{
${ }^{1}$ a commercial 3D image analysis software http://www.fei.com/software/avizo3d/

${ }^{2}$ a free, open source image processing package: http://fiji.sc/Fiji
} 
The node mask, node mask with HEVs and node with a white background stacks were all reduced in size by a quarter in the $x$ and $y$ directions. This resulted in the final voxel size being $5.16 \times 5.16 \times 5 \mu \mathrm{m}$. The stack of the node with a white background was saved as an image sequence of jpeg images for use in COMSOL Multiphysics ${ }^{4}$. COMSOL is used to model the fluid flow. A small area of grey voxels of approximately $80 \mu \mathrm{m}$ in diameter was created on opposite sides of the node, approximately at the position where the afferent and efferent lymphatics entered and exited the node. This was necessary so that boundaries existed for the application of the afferent and efferent lymphatic boundary conditions, described in Section 2.2.

Scan $I \mathrm{P}^{5}$ was used to create a mesh from the edited white and grey image stack. Using the threshold tool, three masks were created. The first one selected only the pale grey voxels that represent the HEVs, the second only the white voxels, and the third only selected the grey voxels that represent the afferent and efferent lymphatic boundary. The afferent and efferent lymphatic mask was dilated by 1 voxel in all directions to create surfaces that represent the afferent and efferent lymph vessels. The three masks that resulted were then added to the model. The model was configured to create a COMSOL mesh file using +FE Free volume meshing. The advanced parameters, such as mesh size, target error and rate of change of element size, were modified. Three mesh sizes were used for the mesh refinement study. For all meshes the target minimum edge length was set to $8.8 \mu \mathrm{m}\left(=\sqrt{5.16^{2}+5.16^{2}+5^{2}} \mu \mathrm{m}\right.$, the longest length between voxels). The maximum length was $8.8,17.6$ or $35.2 \mu \mathrm{m}$ for the three different meshes. The target maximum error was set to $4.4 \mu \mathrm{m}$, half the minimum edge length. The size of the mesh elements on the surface was allowed to change rapidly (set to 75 in ScanIP), because the manual segmentation of the node outline meant a high level of detail was not appropriate. The volume elements within the node changed more slowly (set to 30 ). All other settings were left as default.

\subsection{Model Implementation}

The fluid is modelled as incompressible flow with dynamic viscosity, $\mu, 1.5 \mathrm{cP}$ and density, $\rho$, $1000 \mathrm{~kg} / \mathrm{m}^{3}$ using Darcy's law, i.e., using

$$
\boldsymbol{u}=-\frac{\kappa}{\mu} \nabla p, \quad X \in \Omega
$$

where $\boldsymbol{u}$ is the fluid velocity, $\kappa$ is the interstitial permeability ${ }^{6}$, and $p$ is the fluid pressure. The permeability, $\kappa$, was defined based on the images. A linear relation was used to relate the grey scale of the image to the permeability, e.g. the darker the image, the less dense the material and therefore the more permeable it is. This was implemented in COMSOL using the Matlab Livelink application. A script was written to import the jpeg images for the node on the white

\footnotetext{
${ }^{4}$ A commercial finite element software: $\underline{h t t p: / / w w w . c o m s o l . c o m / ~}$

${ }^{5}$ a commerical software from Simpleware: https://simpleware.com/software/scanip/

${ }^{6}$ an extrinsic value that is a property of the porous media, independent of the fluid
} 
background into the COMSOL Model. The images were imported as functions, called imi where $i$ was the number of the image in the stack. This linear relation has the general form,

$$
\begin{gathered}
\left(-k_{0} \operatorname{im} 1(x, y)+k_{1}\right) \times(z<5 \mu m)+\left(-k_{0} \operatorname{im} 2(x, y)+k_{1}\right) \times(z \geq 5 \mu m) \\
\times(z<10 \mu m)+\cdots+\left(-k_{0} \operatorname{imn}(x, y)+k_{1}\right) \\
\quad \times(z \geq 5(n-1) \mu m), \quad X \in \Omega,
\end{gathered}
$$

212

213

214

215

216

217

218

219

220

221

222

223

224

225

226

227

228

229

230

231

232

233

234

235

236

237

238

239 where $k_{0}$ and $k_{1}$ are constants that define the linear relation between the grey scale of the image and the permeability, $x$ and $y$ are the coordinates of the point in the image which is to be evaluated, $z$ is the distance along the vertical axis and $n$ is the total number of images. Each image is defined on a $5 \mu \mathrm{m}$ thickness using a logical expression, e.g. $(z \geq 5 \mu m) \times$ $(z<10 \mu \mathrm{m})$. Due to file type, the image stack required vertical flipping to correlate with the mesh stack.

The afferent lymphatic flow rate, $F_{\text {in }}$, was given in units of $\mu \mathrm{L} / \mathrm{min}$ in Adair and Guyton (1985). Thus for this modelling study it was divided by $6 \times 10^{7}(\mathrm{~kg} \cdot \mathrm{min} / \mu \mathrm{L} \cdot \mathrm{s})$ (i.e. $60 \mathrm{~min} / \mathrm{s}$ multiplied by $1 \times 10^{6} \mathrm{~kg} / \mu \mathrm{L}$, since the density of the fluid was assumed to be $1 \times 10^{3} \mathrm{~kg} / \mathrm{m}^{3}$ ) to change units to $\mathrm{kg} / \mathrm{s}$. The efferent lymphatic pressure was set to $0 \mathrm{mmHg}$ i.e. efferent lymphatic pressure is considered a gauge pressure. Starling's principle was used for the boundary condition on the HEVs,

$$
J_{v}=L_{p} S\left[\left(p_{c}-p_{i}\right)-\sigma\left(\pi_{c}-\pi_{i}\right)\right]
$$

where $J_{v}\left(\mathrm{~m}^{3} / \mathrm{s}\right)$ is the volume filtered per unit time, $L_{p}(\mathrm{~m} / \mathrm{sPa})$ is the hydraulic conductivity of the blood vessel wall, $S\left(\mathrm{~m}^{2}\right)$ is the surface area of the blood vessel, $p_{c}(\mathrm{~Pa})$ is the pressure in the blood vessel, $p_{i}(\mathrm{~Pa})$ is the pressure in the interstitium, $\sigma$ (no units) is the osmotic reflection coefficient, $\pi_{c}(\mathrm{~Pa})$ is the colloid osmotic pressure in the blood vessel and $\pi_{i}(\mathrm{~Pa})$ is the colloid osmotic pressure in the interstitium. Thus the boundary condition is given by,

$$
\widehat{n_{\text {hev }}} \cdot \boldsymbol{u}=J_{\text {vel }}=\frac{J_{v}}{S}=L_{p}\left[\left(p_{v}-p\right)-\sigma(\Delta \pi)\right], \quad X \in \Gamma_{\text {hev }},
$$

where $\widehat{n_{h e v}}$ is the vector normal to the surface of the HEVs pointing into the node, $J_{v e l}(\mathrm{~m} / \mathrm{s})$ is the fluid flux per unit area across the boundary, $p_{v}(\mathrm{~Pa})$ is the venous pressure, $p(\mathrm{~Pa})$ is the interstitial fluid pressure in the node, and $\Delta \pi=\pi_{v}-\pi_{n}(\mathrm{~Pa})$ where $\pi_{v}(\mathrm{~Pa})$ is the plasma colloid osmotic pressure in the HEVs, and $\pi_{n}(\mathrm{~Pa})$ is the node colloid osmotic pressure; $\sigma$ is set to be equal to 0.9 based on Levick (2009).

The condition on the afferent lymphatic boundary is given by

$$
\widehat{n_{\text {in }}} \cdot \boldsymbol{u}=\frac{F_{\text {in }}}{A_{\text {in }}}, \quad X \in \Gamma_{\text {in }},
$$


where $\widehat{n_{l n}}$ is the vector normal to the afferent lymphatic boundary pointing into the node, $F_{\text {in }}$ is the afferent lymphatic flow rate, set as the experimental value from Adair and Guyton (1985) and $A_{i n}$ is the area of the afferent lymphatic boundary. The pressure at the efferent lymphatic, $p_{\text {out }}$, was defined by the experimental values from Adair and Guyton (1985) and the condition on the efferent lymphatic boundary is given by,

$$
p=p_{\text {out }}, \quad X \in \Gamma_{\text {out }} .
$$

A no flux boundary condition was applied to all other boundaries, $\partial \Omega$. The boundary conditions are summarised in Figure 2. A summary of the parameters used in the model and their approximate values from the literature are shown in Table 1.

\subsection{Mesh Refinement Study}

A mesh refinement study was carried out to optimise the accuracy of the results as a function of the computational cost, such as time and computer memory usage. A coarser mesh, a mesh with fewer elements, produces less accurate results when compared to a fine mesh, which has more elements, however the coarser mesh requires less computational resources.

For the mesh refinement study, the afferent lymphatic flow rate was defined as $7.58 \times 10^{-7}$ $\mathrm{kg} / \mathrm{s}$, the efferent lymphatic pressure was set to $0 \mathrm{~Pa}$ and the blood pressure was $1067 \mathrm{~Pa}$, all these values were taken from the experimental data for one node from Adair and Guyton (1985). The hydraulic permeability of the HEVs was $3.2 \times 10^{-10} \mathrm{~m} /(\mathrm{Pa} \cdot \mathrm{s})$ (Renkin and Michel, 1984) and the colloid osmotic pressure difference was $267 \mathrm{~Pa}$. The experimental data was measured to an accuracy of $0.1 \mu \mathrm{L} / \mathrm{min}$, which is equivalent to $1.67 \times 10^{-9} \mathrm{~kg} / \mathrm{s}$ (Adair and Guyton, 1985).

Figures $3 a$ and $3 c$ show that the efferent lymphatic flow rates between the coarse and fine meshes are smaller than one standard deviation from the mean of the experimental data. Figures $3 b$ and $3 d$ show a comparison between the pressures calculated at the afferent lymphatic boundary for each mesh size. The experimental data were accurate to $0.1 \mathrm{mmHg}$, equivalent to $13 \mathrm{~Pa}$. The differences between the pressures for the three meshes is always below $10 \mathrm{~Pa}$, which also suggests that the coarsest mesh was sufficient to capture the relevant information within the accuracy of the experimental measurements. Therefore, it was concluded that the results obtained from the coarse mesh were suitable for all further analyses. Adopting the coarse mesh enabled calculations over a wider range of parameters within a reasonable time frame, without compromising on accuracy within the context of the experimental data available.

\subsection{Parameter Optimisation}

In order to estimate the values that best fit the data of Adair and Guyton (1985) for the average permeability, $\kappa_{a}$, hydraulic conductivity, $L_{p^{\prime}}$ and colloid osmotic pressure difference, $\Delta \pi$, we 
used a Kriging algorithm. Kriging is an iterative method that interpolates known function values to estimate an optimal parameter set (Krige, 1951). Kriging was implemented in Matlab (Forrester et al., 2008) and was run using the COMSOL with Matlab LiveLink application. Initially arbitrary values for the parameters were tested in order to approximate appropriate bounds used within the Kriging algorithm. Three values were chosen based on these initial tests for each of the parameters, $\kappa_{a}, L_{p}$ and $\Delta \pi$. From these, 27 sample points (27 sets of three parameter values) were estimated in COMSOL and they provided the initial known function values for the objective function in the Kriging algorithm. The error/objective function, $E$, used two least squares terms; one for the afferent lymphatic pressure and the other for the efferent lymphatic flow rate. Thus $E$ was calculated as,

$$
E=\sum_{j=1}^{6} \frac{\left(p_{\text {in }}^{j}-\overline{p_{\text {tn }}^{J}}\right)^{2}}{\left(s_{p}^{j}\right)^{2}}+\sum_{j=1}^{6} \frac{\left(F_{\text {out }}^{j}-\overline{F_{\text {out }}^{J}}\right)^{2}}{10\left(s_{f}^{j}\right)^{2}}
$$

where $p_{\text {in }}^{j}$ is the experimental mean afferent lymphatic pressure at the $j^{\text {th }}$ efferent lymphatic pressure, $p_{i n}^{j}$ is the afferent lymphatic pressure calculated by the model for the same efferent lymphatic pressure, $s_{p}^{j}$ is the experimental standard deviation afferent lymphatic pressure at the $j^{\text {th }}$ efferent lymphatic pressure, $F_{i n}^{j}$ is the experimental mean efferent lymphatic flow rate for the $j^{\text {th }}$ efferent lymphatic pressure, $F_{i n}^{j}$ is the efferent lymphatic flow rate calculated by the model at the same efferent lymphatic pressure, and $s_{f}^{j}$ is the experimental standard deviation for the efferent lymphatic flow rate at the $j^{\text {th }}$ efferent lymphatic pressure. The aim of the Kriging algorithm was to minimise the objective function over a range of values of $L_{p^{\prime}} \Delta \pi, \kappa_{a}$, thus minimising the difference between the experimental data and the model results. Both the afferent lymphatic pressure and the efferent lymphatic flow rate were used because the three parameters being optimised affect the afferent lymphatic pressure and efferent lymphatic flow rate differently. The hydraulic permeability of the blood vessels, $L_{p^{\prime}}$ and the colloid osmotic pressure difference, $\Delta \pi$, had only a small effect on the afferent lymphatic pressure, but a large effect on efferent lymphatic flow rate. However, the average permeability, $\kappa_{a}$, had a large effect in the afferent lymphatic pressure and a small effect on the efferent lymphatic flow rate. Two optimisation methods within the Kriging algorithm were then used to estimate the minimum function value for $E$. Initially, a predicted function was interpolated from the known function values. The first method (local optimisation) found the minimum point of the predicted function. The second method (global optimisation) found the point at which the predicted function had the maximum estimated error. These two new points were estimated by the COMSOL model and then the new function values were interpolated with all of the existing points to update the predicted function (one iteration). The number of iterations for the Kriging algorithm was fixed due to the computational time required. Each iteration required approximately 30 minutes run time and the computational time for the entire process was approximately 40 hours. This allowed 81 total sample points to be estimated. The value of $E$ was found to converged within the number of iterations. 
Adair et al., (1982) hypothesised that the increase in protein concentration in the interstitium of the lymph node was $98 \%$ as a result of fluid transfer. If we assume that $100 \%$ of the increase in protein concentration in the interstitium is due to fluid transfer from the interstitium into the blood vessels, this implies that the blood vessels within the node must absorb fluid, in order to increase the concentration of protein in the lymph node interstitium. This hypothesis required $J_{v e l}<0$ for which, a further assumption that colloid osmotic pressure difference should be greater than the mean venous pressure is required. The venous pressure was assumed to be $973 \mathrm{~Pa}$, the mean venous pressure measured by Adair et al., (1985). In order for $J_{v e l}<0$, it can be seen from Starling's equation that $\left(p_{v}-p\right)-\sigma(\Delta \pi) \leq 0$, and therefore $\left(p_{v}-p\right) \leq \sigma(\Delta \pi)$. Considering the limit where $p=0$ and $\sigma=1, p_{v} \leq \Delta \pi$. Therefore, $\Delta \pi \geq 973 \mathrm{~Pa}$. In Adair et al (1982), the mean equilibrium colloid osmotic pressure difference (when there was no net flow across the blood vessel walls) from eight dog lymph nodes was calculated as $1187 \pm 279 \mathrm{~Pa}$, which showed $\Delta \pi \geq 973 \mathrm{~Pa}$ to be a reasonable assumption.

\subsection{Varying Lymph Node Tissue Permeability}

The grey scale variations in the images contained information about the density of the material within the lymph node. Therefore, these variations can be used as an indicator of permeability, i.e. the lighter the grey scale the denser the material, the lower the permeability. This was integrated into the model by relating the grey scale to permeability, $\kappa$, with a linear relation, equation (2).

The average grey scale of the lymph node images, $G$ (no units), was found using Matlab. After finding the average permeability, $\kappa_{a}\left(\mathrm{~m}^{2}\right)$, the grey scale was related to the permeability by a linear relation that passed through the points $\left(G, \kappa_{a}\right)$ and $\left(1,10^{-14} \mathrm{~m}^{2}\right)$. This was the maximum gradient. The medium gradient was a linear relation that passed though $\left(G, \kappa_{a}\right)$ and $\left(1,\left(\kappa_{a}+10^{-14}\right.\right.$ $\left.\mathrm{m}^{2}\right) / 2$ ). The parameters used are shown in Table 2 and the relations are shown in Figure 4 . With this implementation, it was now possible to examine the flow through the lymph node for two different distributions of the permeability and yet ensure that the average permeability was consistent with the results obtained using the Kriging procedure (and therefore the experimental data).

\section{Results, Discussion and Conclusion}

For the parameter estimation using the Kriging algorithm, the error value, $E$, given by equation (7) was used to evaluate the goodness of fit between the experimental data and the model results. The parameter set which resulted in the lowest error value, $E_{\text {min }}$, was selected as the optimal parameter set for the data. From the Kriging algorithm, with the assumption $\Delta \pi \geq 973$ $\mathrm{Pa}$, it was found that the best fit resulted when $\Delta \pi=973 \mathrm{~Pa}$ with $E_{\text {min }}=15.8$. The results for the WT node in Figure 5 show that although there is good agreement for the afferent lymphatic pressure, the efferent lymphatic flow has a much shallower gradient than is observed in the experiments. 
Therefore we relaxed the assumption that $\Delta \pi \geq 973 \mathrm{~Pa}$ to $\Delta \pi>0$, i.e. the osmotic pressure difference had to be positive. With this relaxed condition, the model produced results that are similar to the experiments, see Figure 6 . This shows that the results from our model and the experimental data are not consistent with the hypothesis in Adair et al., (1982), because fluid extravasation across the HEVs occurs at lower outlet pressures, not absorption. The optimal value of $\Delta \pi=341 \mathrm{~Pa}$ was found by the model, with an error value of $E_{\min }=9$. The gradient of the efferent lymphatic pressure to efferent lymphatic flow rate was more similar to the experimental data. The average permeability of the lymph node tissue, $\kappa_{a}=3.8 \times 10^{-11}$, was $5.2 \%$ higher than the $\Delta \pi \geq 973 \mathrm{~Pa}$ limited case and the hydraulic conductivity of the HEVs was $149 \%$ higher but the same order of magnitude, $5.1 \times 10^{-8} \mathrm{~m} / \mathrm{Pa} \cdot \mathrm{s}$ compared to $2 \times 10^{-8}$ $\mathrm{m} / \mathrm{Pa} \cdot \mathrm{s}$. For the PLT node, the Kriging algorithm was also used for $\Delta \pi>0$, with $E_{\min }=9$, the results can be seen in Figure 7. The values for $\kappa_{a}$ and $L_{p}$ were similar to the WT node, the value of $\Delta \pi, 553 \mathrm{~Pa}$, for the PLT node is about $200 \mathrm{~Pa}$ higher than for the WT node.

Fluid flow stream tubes are plotted in Figure 8 to show the fluid flux through the node for the different efferent lymphatic pressures. Stream tubes are curves that the velocity vectors are tangential to and have an area which was defined, in this case, as proportional to the fluid flux. For the highest efferent lymphatic pressure, $2170 \mathrm{~Pa}$, there are fewer stream tubes at the efferent lymphatic than the node for the lowest efferent lymphatic pressure, $0 \mathrm{~Pa}$.

The value of $J_{\text {vel }}$ changed for the different efferent lymphatic pressures. For the two lowest pressures, the flow direction was out of the HEVs. For the other four pressures, the flow was into the vessels. This implied that for some efferent lymphatic pressures $270 \mathrm{~Pa}<p_{\text {out }}<697 \mathrm{~Pa}$ part of the fluid would be absorbed by the HEVs and part would be extravasated. It can be shown that this occurs for all the efferent lymphatic pressures used in this study, see Figure 9. The red HEVs surfaces are releasing fluid from the blood vessels and into the lymph node, whereas the blue surfaces are absorbing fluid. For efferent lymphatic low pressures, Figures 9a and $9 \mathrm{~b}$, the majority of HEVs are extravasating fluid into the node. For higher efferent lymphatic pressures, Figures 9c and 9d, the majority of HEVs are absorbing fluid. Two varying permeability cases were modelled and compared to the constant case. The results show that the inclusion of a gradient for the permeability caused more flow through the centre of the node and less around the outside, as seen in Figures 10 and 11 . The varying permeability cases slightly lowered the flow rate for each efferent lymphatic pressure. This implied that there was increase fluid reabsorption across HEVs. This was a result of the higher permeability values for the node tissue, as shown in Figure 12.

The average permeability of the interstitium was estimated as $10^{-11} \mathrm{~m}^{2}$ by optimising the image based model of a mouse lymph node to the experimental data from canine lymph nodes (Adair et al., 1985). This permeability was comparable to that of mouse tail skin, blood clot and mesentery (Swartz and Fleury, 2007). Relating the permeability linearly to the grey scale resulted in more flow through the centre of the node than the constant permeability case. The images used to create the model did not clearly show some of the structural detail that has been described in the literature. Within the lymph node, beneath the capsule is the subcapsular 
sinus (Ohtani and Ohtani, 2008). This is thought to be an area of low resistance to the flow, however it was not clearly shown in the images used for this study. To resolve this issue a follow up study using high resolution micro computed tomography images, which show more structural detail, is required.

The values of $L_{p}$ found as part of the parameter optimisation were three orders of magnitude higher than values for capillaries found in Renkin and Michel (1984). Since only the HEVs were modelled, the value of $L_{p}$ had to be higher, the vessels must allow more fluid to cross the wall, so that the same amount of flow across the blood vessel wall in the experiment, which will have many more blood vessels, could be achieved by the model

Initially, it was assumed that $J_{v e l}<0$ and therefore $\Delta \pi \geq 973$ Pa due to the hypothesis by Adair et al., (1982) that the increase in protein concentration was caused by the fluid being absorbed into the HEVs. However, it was possible to obtain a much better fit, $E_{\min }=9$ compared to $E_{\min }=15$, for the model when $\Delta \pi \geq 0$ was compared to experimental data. Hence, the original assumption that $\Delta \pi \geq 973$ was inaccurate. However, the parameter fitting had been able to account for most of the behaviour of the efferent lymphatic flow rate as the efferent lymphatic pressure increases, implying that the fluid flow dominates this behaviour. It was not able to reproduce the curve of the efferent lymphatic pressure verses efferent lymphatic flow rate. In a follow-on study the model will be developed to include a convection/diffusion expression of the protein transport to allow investigation of the colloid osmotic pressure which it was not possible to capture in the current model. Introducing a convection/diffusion model for proteins will also introduce a time-dependent variable to the model, which will allow investigation of transient effects. However, the present model is the first necessary step for capturing the main profiles of the afferent to efferent lymphatic pressure relations and the efferent lymphatic flow rate to efferent lymphatic pressure. The model presented in this paper shows that the fluid flow is the dominant behaviour which agrees with the hypothesis in Adair et al., (1982).

Considering the grouping $L_{p} \sigma \Delta \pi(\mathrm{m} / \mathrm{s})$ and taking the largest value of $L_{p}=6.7 \times 10^{-10}$ $\mathrm{m} /(\mathrm{Pa} \cdot \mathrm{s})$ from Renkin and Michel (1984) and $\sigma=0.9$, the value for the grouping is $L_{p} \sigma \Delta \pi=$ $1.3 \times 10^{-6} \mathrm{~m} / \mathrm{s}$. For the WT node, using the values for the optimised model, this value is $L_{p} \sigma \Delta \pi=1.57 \times 10^{-5} \mathrm{~m} / \mathrm{s}$ and the PLT node, $L_{p} \sigma \Delta \pi=2.16 \times 10^{-5} \mathrm{~m} / \mathrm{s}$. These values found for the optimised models are an order of magnitude larger than calculated using the values from Renkin and Michel (1984) and Levick (2009). In order to make the model values the same order of magnitude as in the literature, the value of $\sigma$ would need to be less than 0.57 for the WT node or 0.42 for the PLT node, implying that the HEVs are more leaky than capillaries. If a lower value of $\sigma$ were used, higher values of $\Delta \pi$ would be required to achieve the same fit to experimental data as was achieved from the Kriging algorithm, meaning that the model values of $\Delta \pi$ could be more similar to the value estimated from Adair and Guyton (1985), 2080 Pa.

The HEVs hydrostatic pressure was fixed at $973 \mathrm{~Pa}$, as calculated as the mean of the venous pressure from the experiments from Adair and Guyton (1985). The lymph node contains arteries, capillaries and veins, so it is expected that the hydrostatic pressure of the blood vessels should vary throughout the node. The pressure in the veins is lower than the pressure in the arteries, 
therefore, if the average blood pressure was found within the node it is likely to be higher than the venous pressure measured. The optimised value of $L_{p}$ found by Kriging is two orders of magnitude higher than the values recorded in literature. If the value of $L_{p}$ from literature was used, the HEVs hydrostatic pressure, $p_{v}$, would need to be increased by two orders of magnitude is order to produce the same $J_{v e l}$ currently implemented in the model.

In conclusion, this paper presents a theoretical and computational framework to create an image based computational model of fluid flow through a lymph node. The model has related the grey scale values of the images to the permeability of the lymph node tissue with two different linear relations and these were compared to a case where the permeability in the node interstitium was constant, showing that the majority of the fluid flow is through the centre of the node.

\section{References}

Adair TH, Guyton AC (1983) Modification of lymph by lymph nodes. ii. Effect of increased lymph node venous blood pressure. American Journal of Physiology: Heart and Circulation Physiology 245:H616-H622

Adair TH, Guyton AC (1985) Modification of lymph by lymph nodes iii. Effect of increased lymph hydrostatic pressure. American Journal of Physiology: Heart and Circulation Physiology 249:H777-H782

Adair TH, Moffatt DS, Paulsen AW, Guyton AC (1982) Quatitiation of changes in lymph protein concentration during lymph node transit. American Journal of Physiology: Heart and Circulation Physiology 243:H351-H359

Beltman JB, Maree AF, Lynch JN, Miller MJ, de Boer RJ (2007) Lymph node topology dictates t cell migration behavior. J Exp Med 204(4):771-80

Bogle G, Dunbar PR (2008) Simulating t-cell motility in the lymph node paracortex with a packed lattice geometry. Immunol Cell Biol 86(8):676-87

Bogle G, Dunbar PR (2010) Agent-based simulation of t-cell activation and proliferation within a lymph node. Immunol Cell Biol 88(2):172-9

Bogle G, Dunbar PR (2012) On-lattice simulation of t cell motility, chemotaxis, and trafficking in the lymph node paracortex. PLoS One 7(9):e45,258

Dixon JB, Greiner ST, Gashev AA, Cote GL, Moore JE Jr, Zawieja DC (2006) Lymph flow, shear stress, and lymphocyte velocity in rat mesenteric prenodal lymphatics. Microcirculation 13 Forrester A, Sobester A, Keane A (2008) Engineering design via surrogate modelling: a practical guide. John Wiley \& Sons

Gretz JE, Norbury CC, Anderson AO, Proudfoot AE, Shaw S (2000) Lymph-borne chemokines and other low molecular weight molecules reach high endothelial venules via specialized conduits while a functional barrier limits access to the lymphocyte microenvironments in lymph node cortex. J Exp Med 192(10):1425-40 
479 Grigorova IL, Panteleev M, Cyster JG (2010) Lymph node cortical sinus organization and 480 relationship to lymphocyte egress dynamics and antigen exposure. Proceedings of the National 481 Academy of Sciences 107(47):20,447-20,452

482 Krige DG (1951) A statistical approach to some basic mine valuation problems on the witwatersrand. Journal of the Chemical, Metallurgical and Mining Society of South Africa

$484 \quad 52: 119-139$

485 Levick JR (2009) An introduction to cardiovascular physiology, 5th edn. London : Hodder Arnold 486 Mayer J, Swoger J, Ozga AJ, Stein JV, Sharpe J (2012) Quantitative measurements in

487 3-dimensional datasets of mouse lymph nodes resolve organ-wide functional dependencies.

488 Comput Math Methods Med 2012:128,431 Ohtani O, Ohtani Y, Carati CJ, Gannon BJ (2003) Fluid and cellular pathways of rat lymph nodes in relation to lymphatic labyrinth and Aquaporin-1 expression. Archives of Histology and

491 Cytology 66:261-272 Ohtani O, Ohtani Y (2008) Structure and function of rat lymoh nodes. Archives of Histology and 493 Cytology 71:69-76

494 Renkin EM, Michel CC (eds) (1984) Handbook of Physiology: Section 2: The Cardiovascular System, vol Volume IV: Microcirculation, Part 1. American Physiological Society Schindelin J, Arganda-Carreras I, Frise E, Kaynig V, Longair M, Pietzsch T, Preibisch S, Rueden C, Saalfield S, Schmid B, Tinevez J-Y, White DJ, Hartenstein V, Eliceiri K, Tomancak P, Cardona A (2012) Fiji: an open-source platform for biological-image analysis. Nature Methods 9:676682Swartz MA, Fleury ME (2007) Intersititial flow and its effect in soft tissues. Annual Review of 500 Biomedical Engineering 9:229-256

501 Tomei AA, Siegert S, Britschgi MR, Luther SA, Swartz MA (2009) Fluid flow regulates stromal 502 cell organization and $\mathrm{ccl} 21$ expression in a tissue-engineered lymph node microenvironment. 503 The Journal of Immunology 183(7):4273-4283

504 


\section{Figure Captions:}

Fig. 1 Figures showing the process of image processing: A) Original 16 bit image. B) Filtered 8 bit image with auto adjusted brightness and contrast. The filtered image results in more distinguishable features. C) Node mask, D) node with white background, E) node mask with HEVs. The stacks were all reduced in size by a quarter in the $x$ and $y$ directions. This made the pixel size $5.16 \mu \mathrm{m}$, which is closer to the $z$ spacing of $5 \mu \mathrm{m}$. B) The node mask with HEVs was used for meshing the geometry. C) The node on the white background was used as the image function in COMSOL. F) Cropped section from lymph node image. The yellow line represents the line along which the grey scale was evaluated, resulting in $\mathrm{G}$ ) the greyscale profile over a HEV.

Fig. 2 Sketch summarising the lymph node model. Dashed black lines show where the afferent and efferent vessels would be, although these are not modelled. The arrows labelled with, $\hat{n}$, show the positive normal vectors to the boundary they are on.

Fig. 3 Comparison of efferent flow rate and afferent pressure for three different meshes for each node. The finest mesh is 1 , the coarsest mesh is 4 . The dotted lines shows the experimental measurement accuracy and the dashed lines show \pm 1 standard deviation, based on the experimental data from four nodes with efferent lymphatic pressure 0 from Adair and Guyton (1985). The solid black line shows the result from the finest mesh. A) Efferent lymphatic flow for WT node, B) Afferent pressure for WT node, C) Efferent lymphatic flow for PLT node, D) Afferent pressure for PLT node.

Fig. 4 Graphs showing permeability to grey scale relations for A) WT and B) PLT node. Solid line shows constant permeability found by parameter fitting.

Fig. 5 Comparison of WT model results for case where $\Delta \pi \geq 973 \mathrm{~Pa}$, circles, to experimental data of four nodes, lines. Error bars show one standard deviation. A) Compares afferent pressure to efferent pressure. There is a good agreement with the experimental data B) Compares efferent flow rate to efferent pressure. The gradient of the model results is too shallow. Parameters found from Kriging: $E_{\min }=15.87$ (no units), $\kappa_{a}=3.5959 \times 10^{-11} \mathrm{~m}^{2}$, $L_{p}=2.0473 \times 10^{-8} \mathrm{~m} / \mathrm{Pa} \cdot \mathrm{s}, \Delta \pi=973 \mathrm{~Pa}$.

Fig. 6 Comparison of WT model results for case where $\Delta \pi \geq 0 \mathrm{~Pa}$, circles, to experimental data of four nodes, lines. Error bars show one standard deviation. A) Compares afferent pressure to efferent pressure. B) Compares efferent flow rate to efferent pressure. Both graphs show good agreement to the experimental data. Green dots show model results. Parameters found from Kriging: $E_{\min }=8.988$ (no units), $\kappa_{a}=3.7825 \times 10^{-11} \mathrm{~m}^{2}, L_{p}=5.1113 \times 10^{-8} \mathrm{~m} / \mathrm{Pa} \cdot \mathrm{s}$, $\Delta \pi=341 \mathrm{~Pa}$. 
Fig. 7 Comparison of PLT model results for case where $\Delta \pi \geq 0 \mathrm{~Pa}$, circles, to experimental data of four nodes, lines. Error bars show one standard deviation. A) Compares afferent pressure to efferent pressure. B) Compares efferent flow rate to efferent pressure. Both graphs show good agreement to the experimental data. Green dots show model results. Parameters found from kriging: $E_{\min }=9.029$ (no units), $\kappa_{a}=3.4515 \times 10^{-11} \mathrm{~m}^{2}, L_{p}=4.3304 \times 10^{-8} \mathrm{~m} / \mathrm{Pa} \cdot \mathrm{s}$, $\Delta \pi=553 \mathrm{~Pa}$.

Fig. 8 Stream tubes for efferent lymphatic pressure $=0 \mathrm{~Pa}$. Scale bar $=250 \mu \mathrm{m}$. The flow rate is higher at the near the afferent and efferent lymphatic boundaries, shown by the clustering of stream lines on either side of the node. The passage of flow through the node is much slower with the majority taking a direct route between the afferent and efferent boundaries.

Fig. 9 Comparison of flow entering and leaving the HEVs for different efferent pressures for PLT node. Only the HEVs are shown. Light areas shows where fluid is leaving the HEVs and entering the node. Dark areas shows where is fluid leaving the node and entering the HEVs. A) Efferent pressure $0 \mathrm{~Pa}$, the majority of HEVs are extravasating fluid. B) Efferent pressure $270 \mathrm{~Pa}$, less of the HEVs are extravasating fluid. C) Efferent pressure $697 \mathrm{~Pa}$, the majority of the HEVs are absorbing fluid. D) Efferent pressure $2170 \mathrm{~Pa}$, almost all of the HEVs are absorbing fluid except a

Fig. 10 Flux percentage difference for WT node. Comparison of the flux percentage difference though different annuli (shown in images below graph) through central 2D plane of lymph node. Values are normalised to constant results for comparison, hence all constant values are 0.

Fig. 11 Flux percentage difference for PLT node. Comparison of the flux percentage difference though different annuli (shown in images below graph) through central 2D plane of lymph node. Values are normalised to constant results for comparison, hence all constant values are 0 .

Fig. 12 Comparison of WT model results for case where $\Delta \pi \geq 0 \mathrm{~Pa}$ and the permeability is related to the greyscale with the maximum gradient. The circles show the model results and the lines the experimental data. Error bars show one standard deviation. A) Compares afferent pressure to efferent pressure. B) Compares efferent flow rate to efferent pressure. Note that efferent flow rate is lower for the maximum gradient compared to the constant gradient. 
Table Captions:

Table 1 Parameters for lymph node model.

579 Table 2 Parameters for varying permeability for nodes.

Tables:

Table 1:

\begin{tabular}{|c|c|c|c|c|}
\hline Parameter & Symbol & Unit & Value & Source \\
\hline $\begin{array}{l}\text { Afferent lymphatic } \\
\text { flow rate }\end{array}$ & $F_{\text {in }}$ & $\mathrm{kg} / \mathrm{s}$ & $7.596 \times 10^{-7}$ & $\begin{array}{l}\text { Mean from experimental data } \\
\text { (Adair and Guyton 1985) }\end{array}$ \\
\hline $\begin{array}{l}\text { Efferent lymphatic } \\
\text { pressure }\end{array}$ & $p_{\text {out }}$ & $\mathrm{Pa}$ & $\begin{array}{c}0,270,697 \\
1103,1653 \\
2170\end{array}$ & $\begin{array}{l}\text { Mean from experimental data } \\
\text { (Adair and Guyton 1985) }\end{array}$ \\
\hline Blood pressure & $p_{v}$ & $\mathrm{~Pa}$ & 973 & $\begin{array}{l}\text { Mean from experimental data } \\
\text { (Adair and Guyton 1985) }\end{array}$ \\
\hline Average Permeability & $\kappa_{a}$ & $\mathrm{~m}^{2}$ & $\begin{array}{l}10^{-14} \text { to } \\
10^{-8}\end{array}$ & $\begin{array}{l}\text { Range from Swartz and Fleury } \\
\text { (2007) }\end{array}$ \\
\hline Varying Permeability & $k_{0}, k_{1}$ & $m^{2}$ & $\begin{array}{c}10^{-14} \text { to } \\
10^{-10}\end{array}$ & $\begin{array}{l}\text { No values in literature. Maximum } \\
\text { value from parameter fitting (see } \\
\text { section } 2.4 \text { ), minimum value from } \\
\text { Range from Swartz and Fleury } \\
\text { (2007) }\end{array}$ \\
\hline Fluid density & $\rho$ & $\mathrm{kg} \cdot \mathrm{m}^{-3} / \mathrm{s}$ & 1000 & \\
\hline Dynamic viscosity & $\mu$ & $\mathrm{Pa} \cdot \mathrm{S}$ & 0.0015 & From Dixon et al. (2006) \\
\hline $\begin{array}{c}\text { Hydraulic } \\
\text { permeability of blood } \\
\text { vessels }\end{array}$ & $L_{p}$ & $\mathrm{~m} /(\mathrm{Pa} \cdot \mathrm{s})$ & $\begin{array}{l}1.02 \times 10^{-11} \\
\text { to } 6.7 \times 10^{-10}\end{array}$ & $\begin{array}{l}\text { Range from Renkin and Michel } \\
{ }^{0}(1984) \text { for capillaries }\end{array}$ \\
\hline $\begin{array}{l}\text { Osmotic reflection } \\
\text { coefficient }\end{array}$ & $\sigma$ & $\begin{array}{l}\text { no } \\
\text { units }\end{array}$ & $\begin{array}{l}0.8 \text { to } 0.95 \\
\text { fixed at } 0.9\end{array}$ & From Levick (2009) \\
\hline $\begin{array}{l}\text { Colloid osmotic } \\
\text { pressure difference }\end{array}$ & $\Delta \pi$ & $\mathrm{Pa}$ & 2080 & $\begin{array}{l}\text { Mean from experimental data } \\
\text { (Adair and Guyton, 1985), } \\
\text { calculated using Navar equation } \\
\text { and protein concentrations of } \\
\text { plasma, afferent and efferent } \\
\text { lymph }\end{array}$ \\
\hline
\end{tabular}


585 Table 2:

\begin{tabular}{ccccc}
\hline & \multicolumn{2}{c}{ WT } & $k_{0}$ & PLT \\
& $k_{0}$ & $k_{1}$ & $k_{1}$ \\
\hline Maximum & $6.2086 \times 10^{-11}$ & $6.2096 \times 10^{-11}$ & $6.8988 \times 10^{-11}$ & $6.8998 \times 10^{-11}$ \\
Medium & $3.1043 \times 10^{-11}$ & $4.9960 \times 10^{-11}$ & $3.4494 \times 10^{-11}$ & $5.1756 \times 10^{-11}$ \\
Constant & 0 & $3.7825 \times 10^{-11}$ & 0 & $3.4515 \times 10^{-11}$ \\
\hline
\end{tabular}

586 

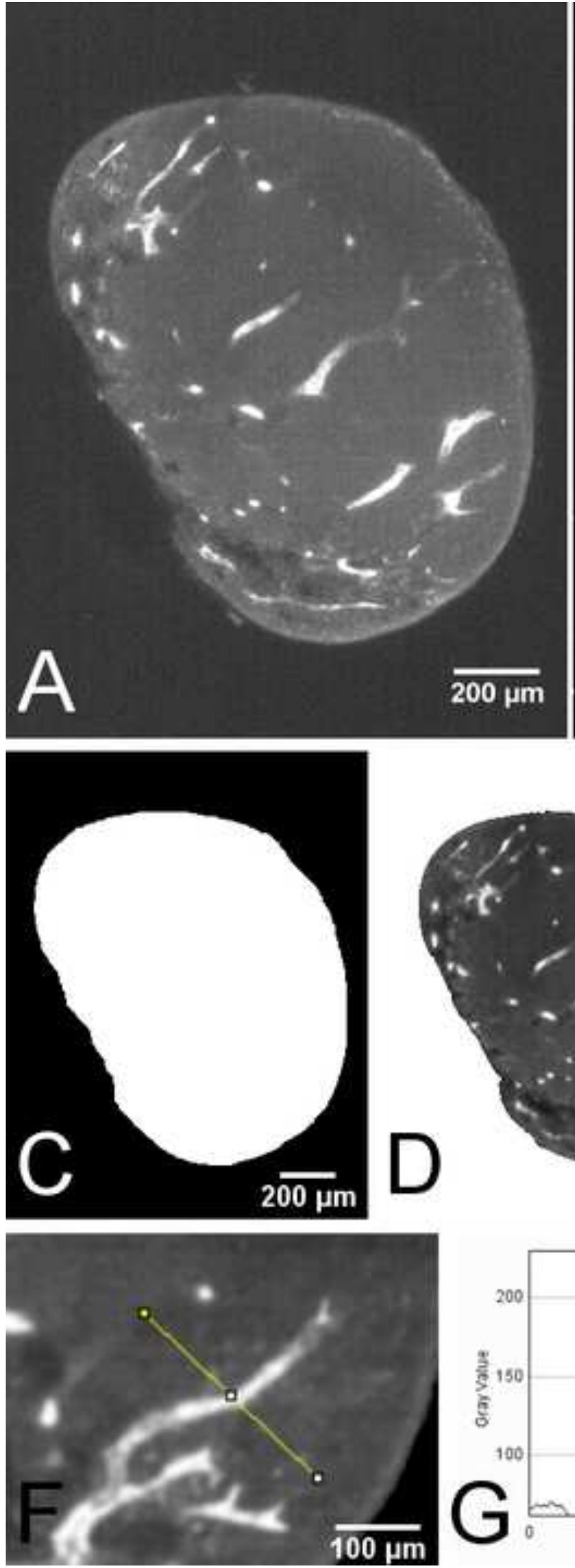
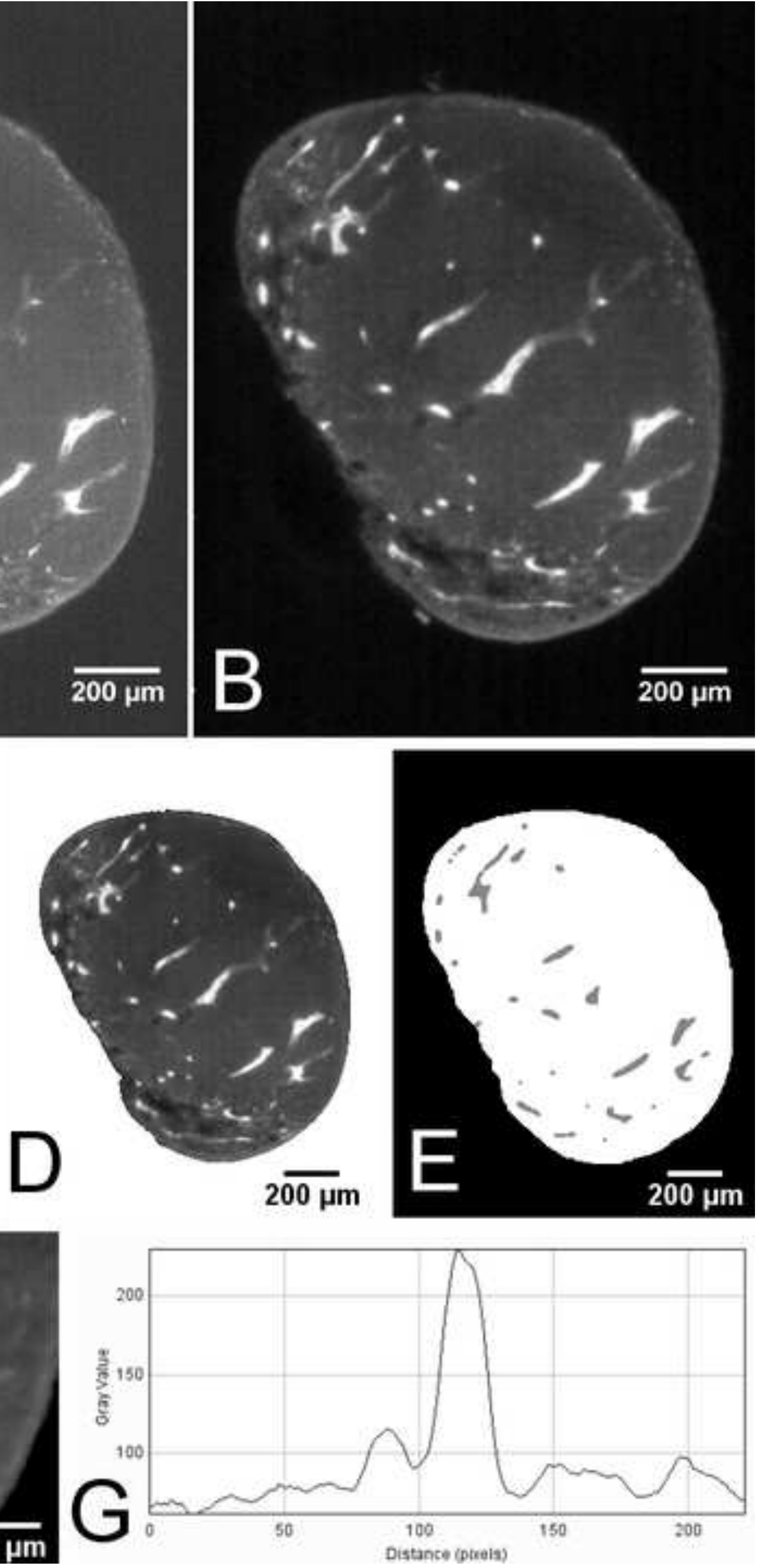


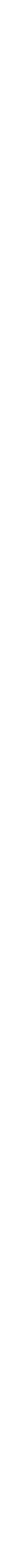




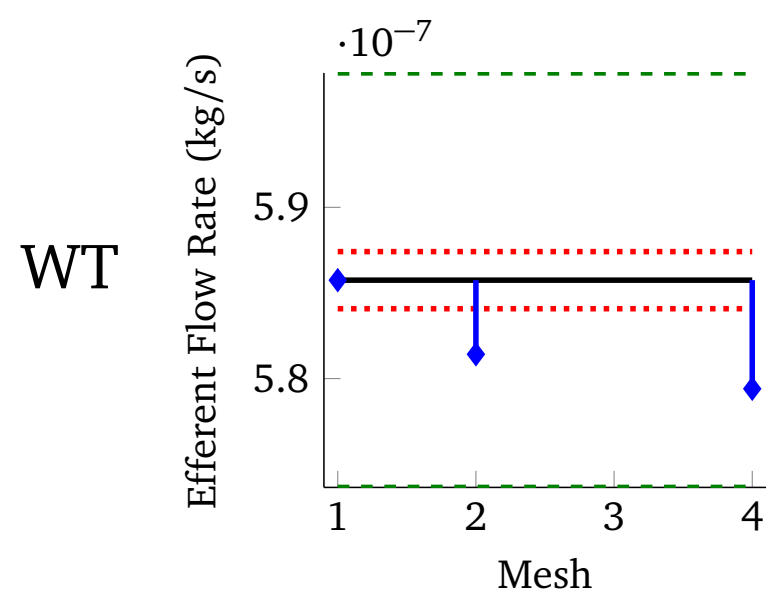

Constant permeability



Medium permeability

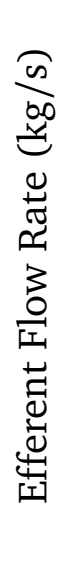

5.6

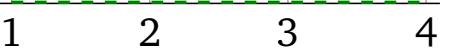

Mesh

Maximum permeability

B)

C)

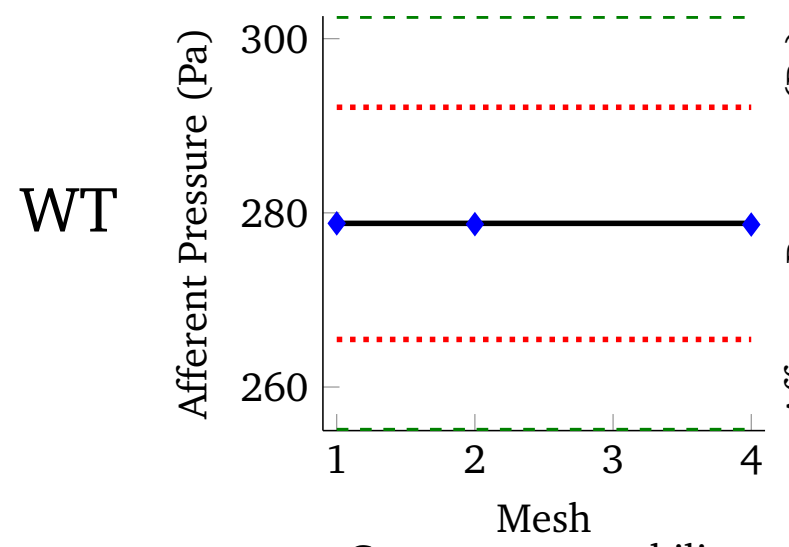

Constant permeability

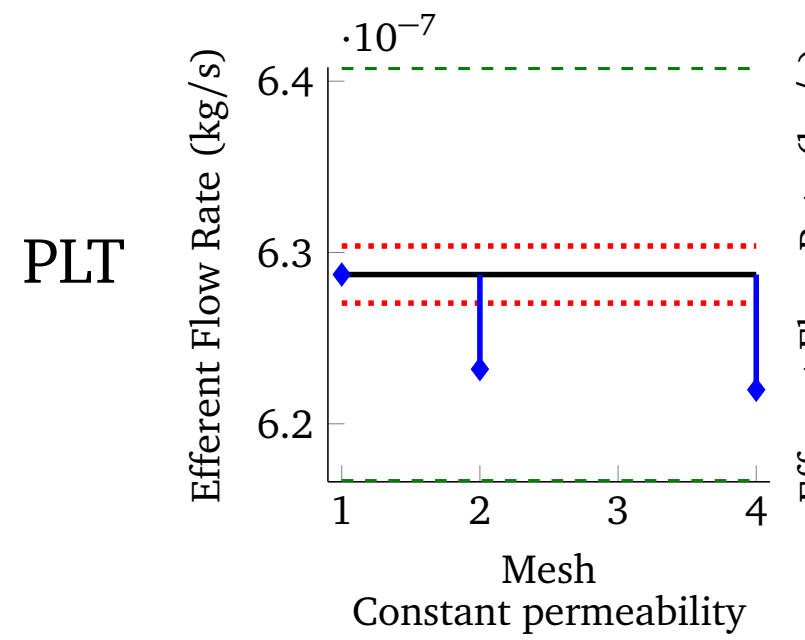

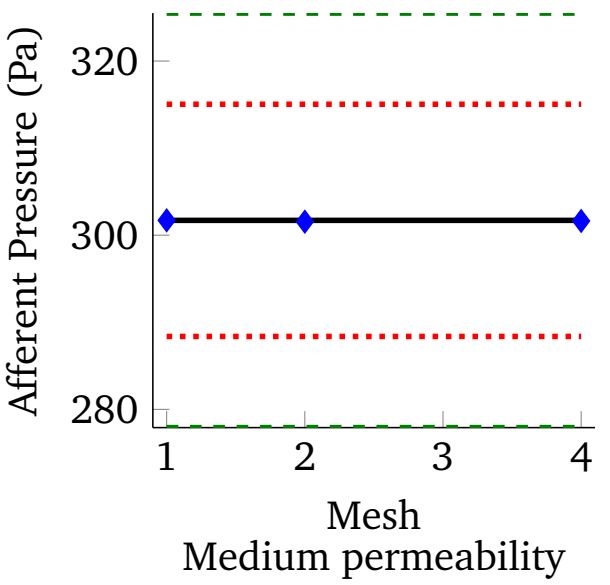
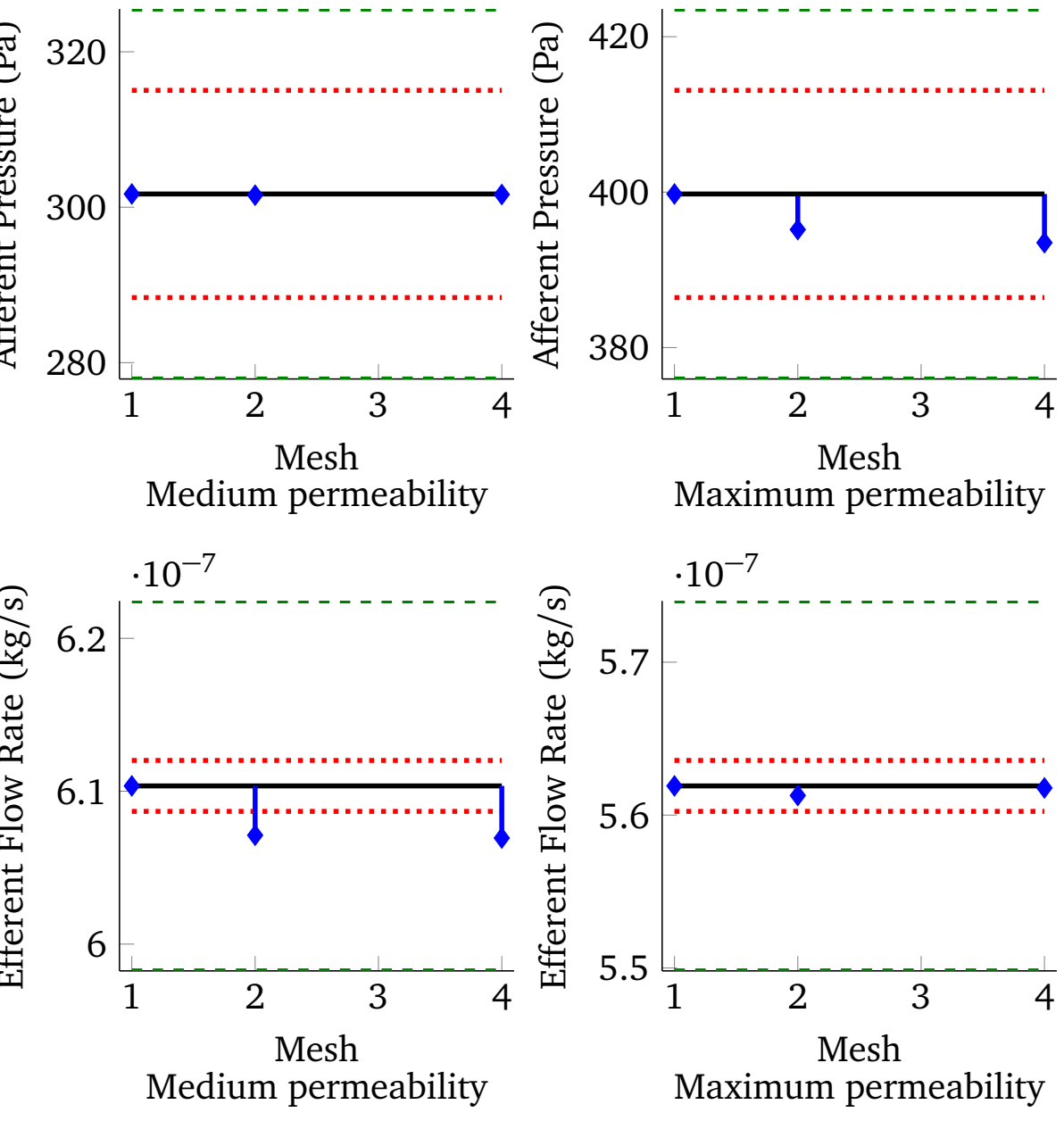

D)

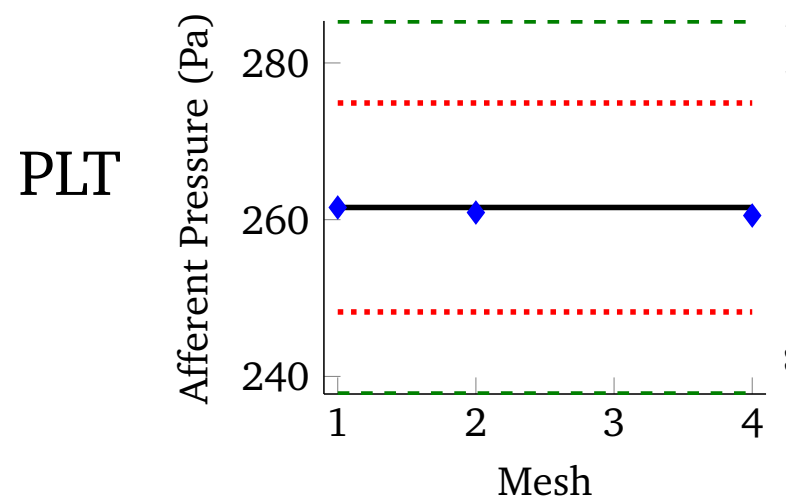

Constant permeability

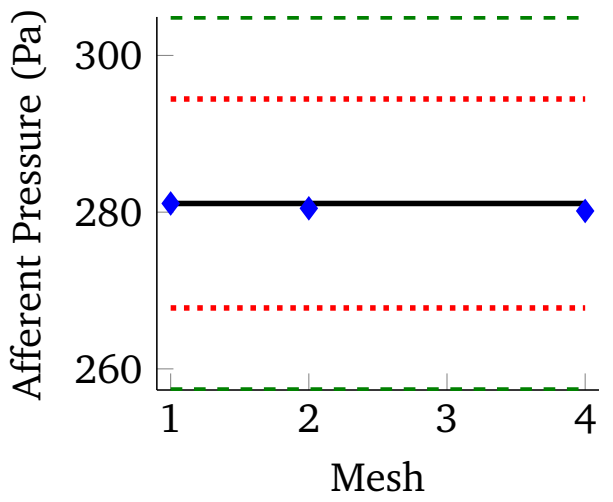

Medium permeability

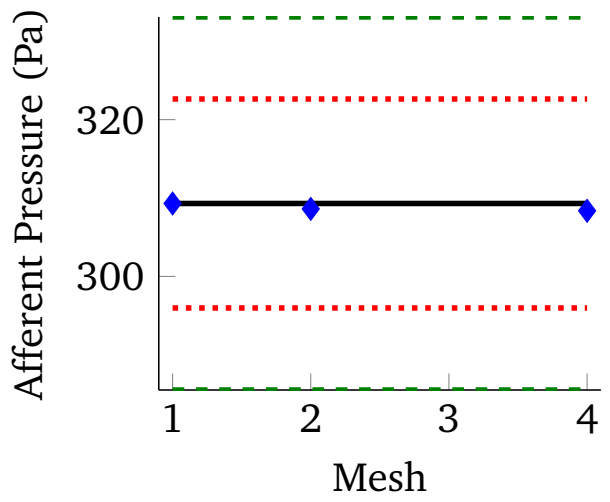

Maximum permeability 
A)

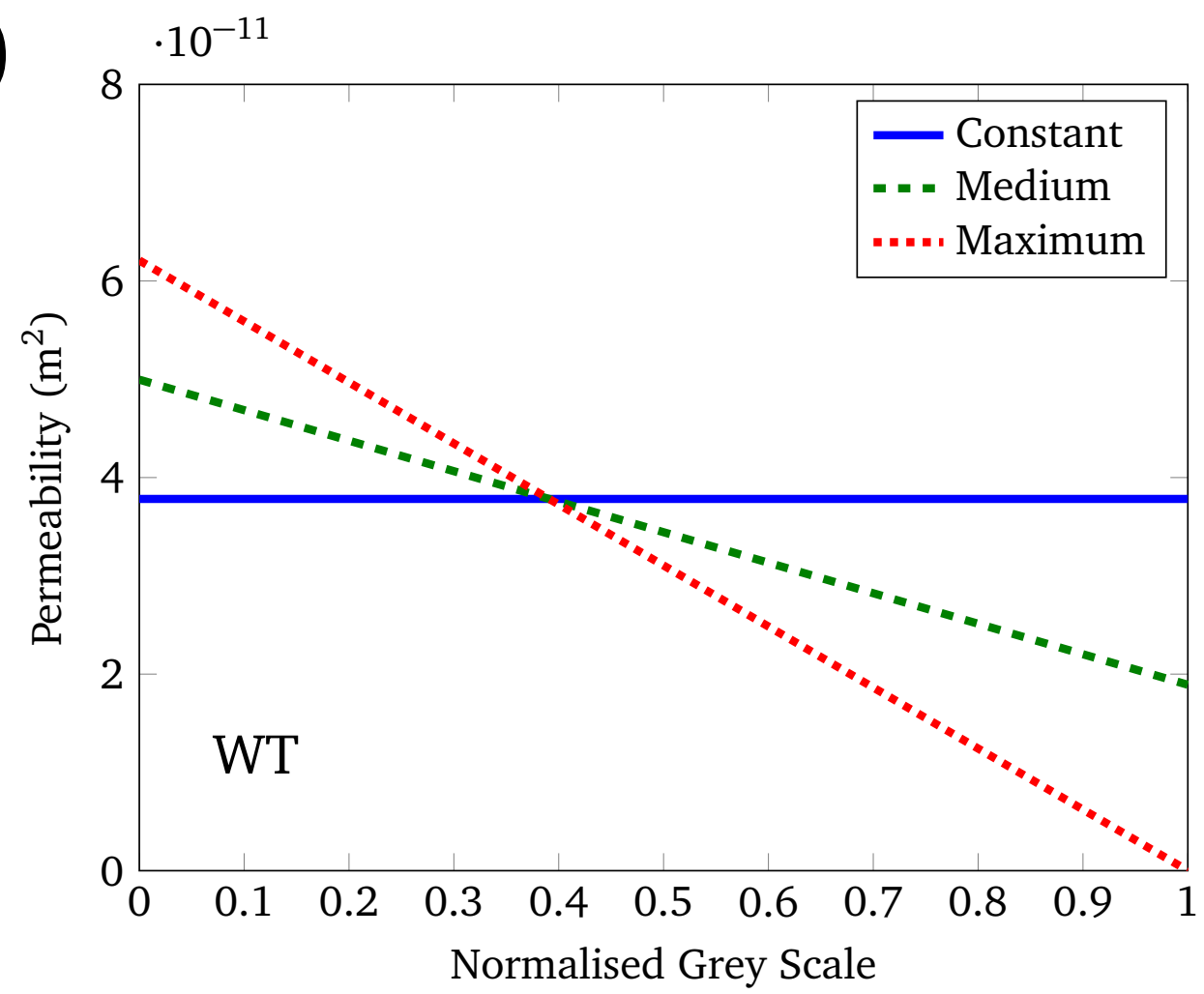

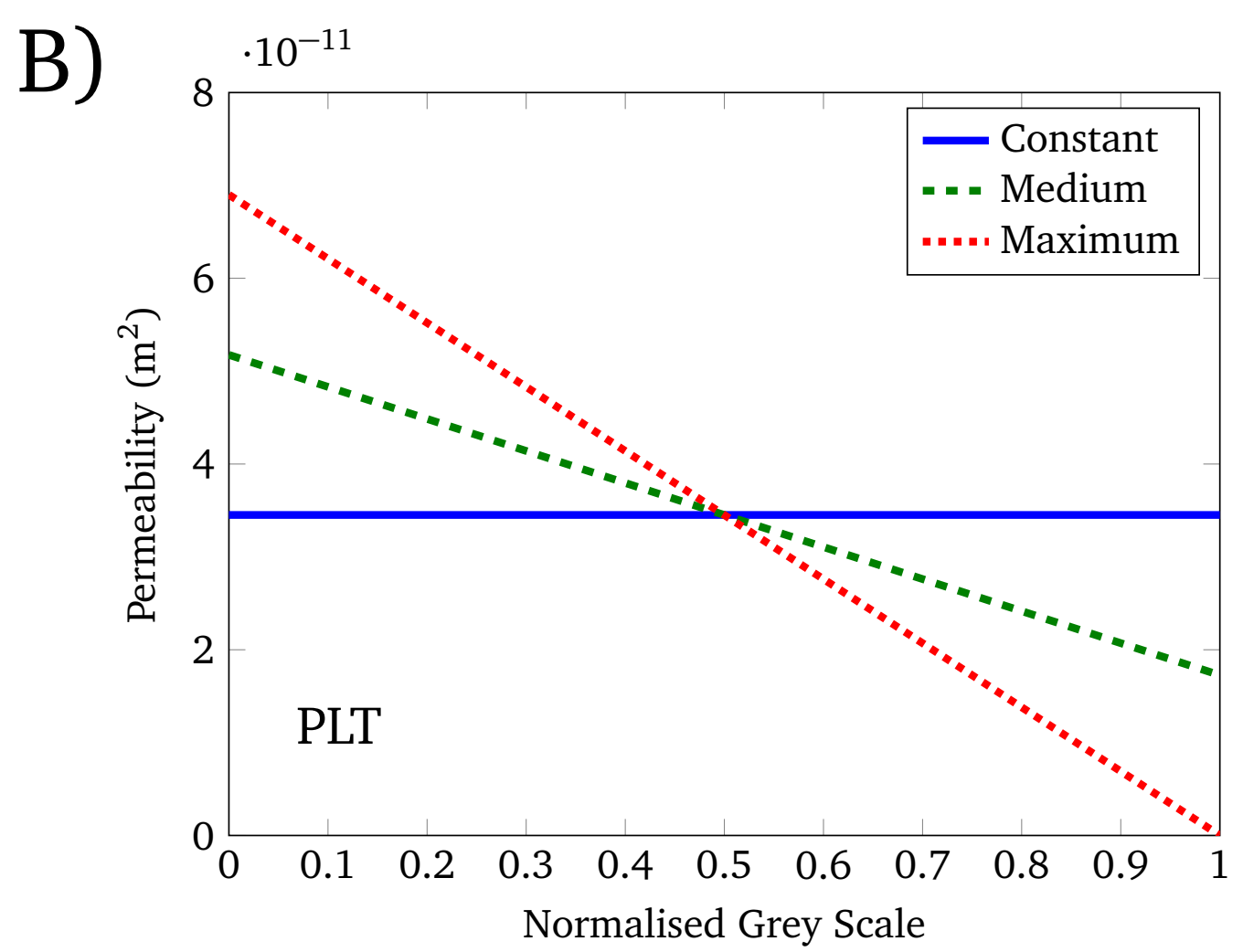


A)

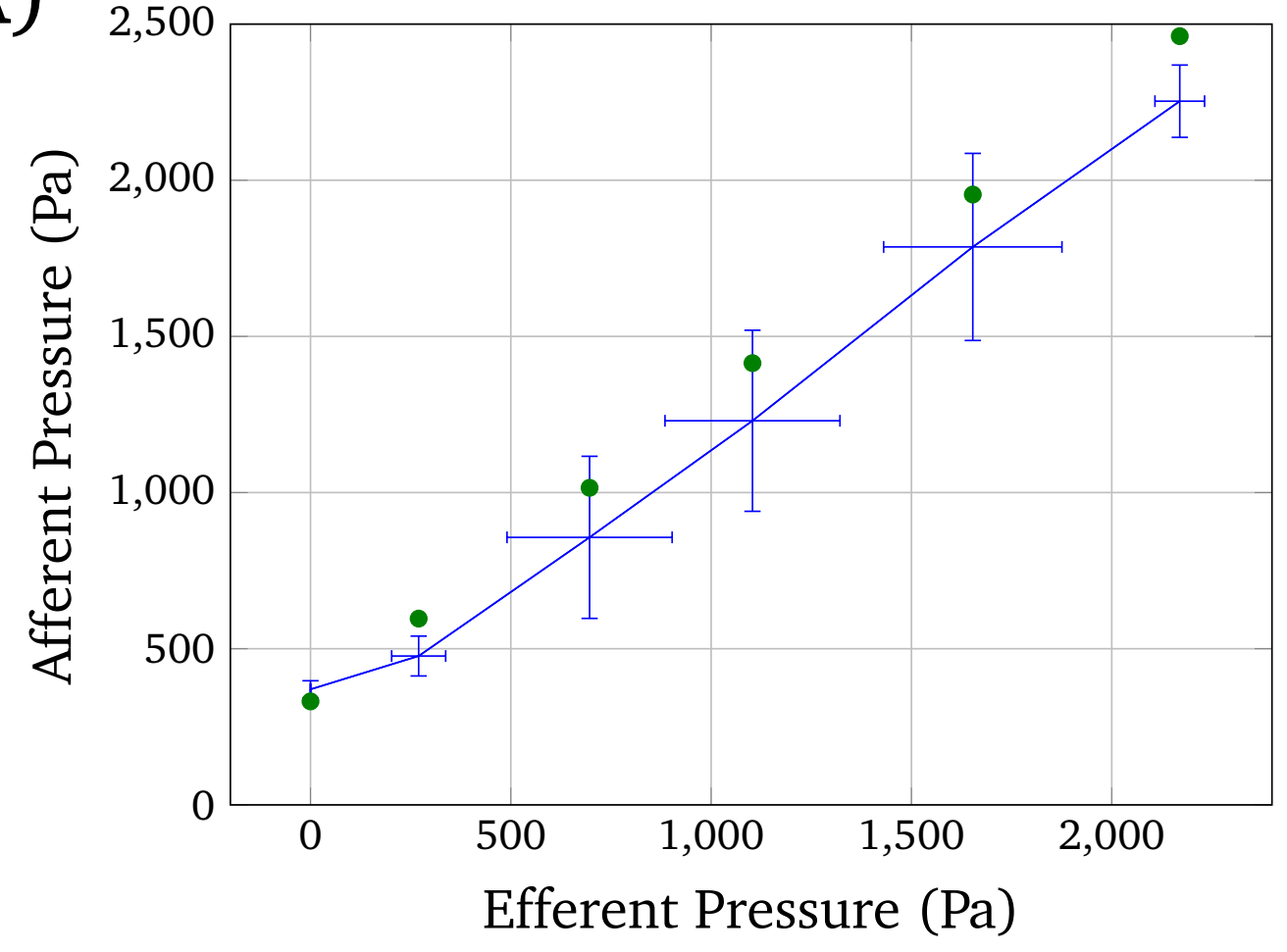

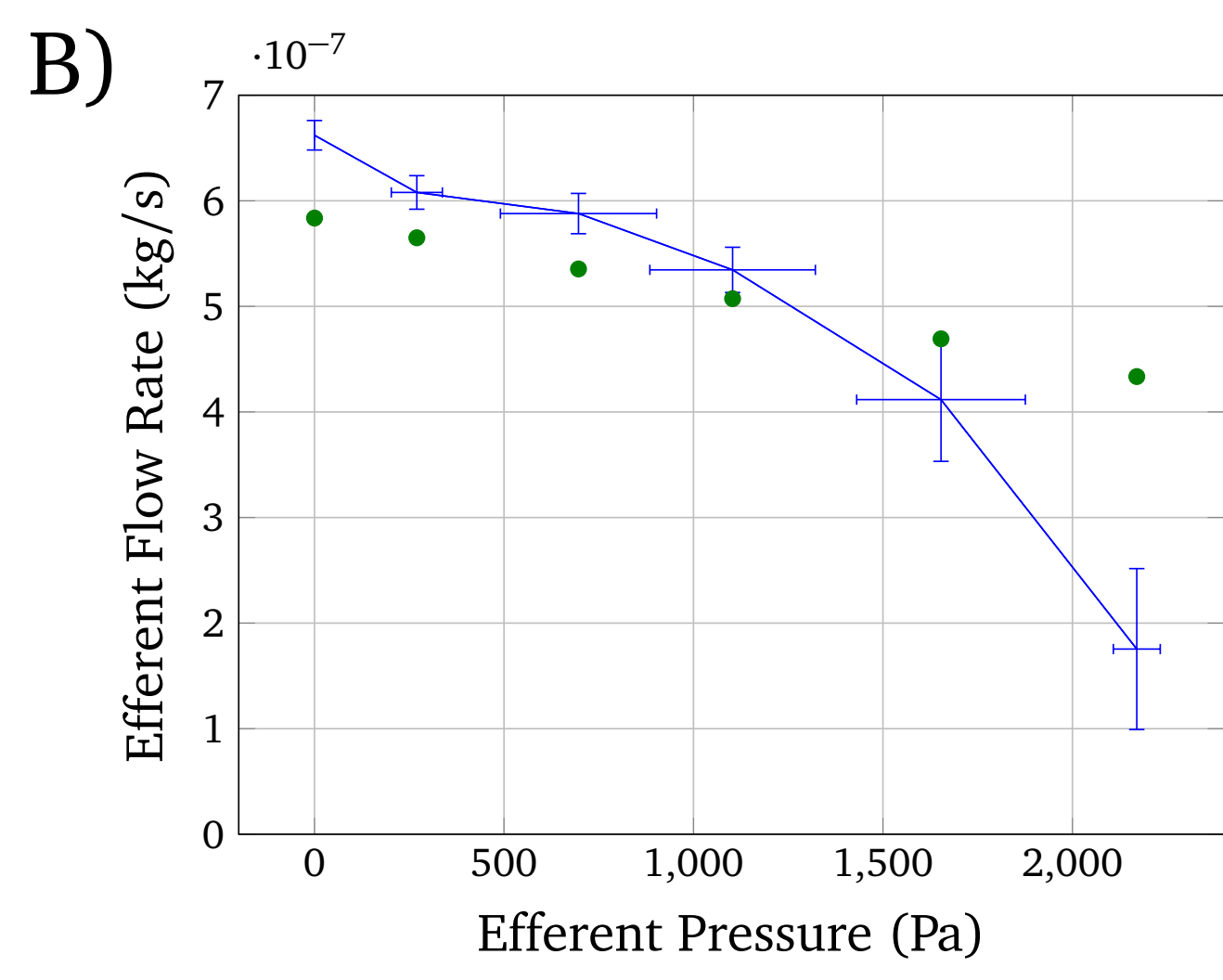


A)

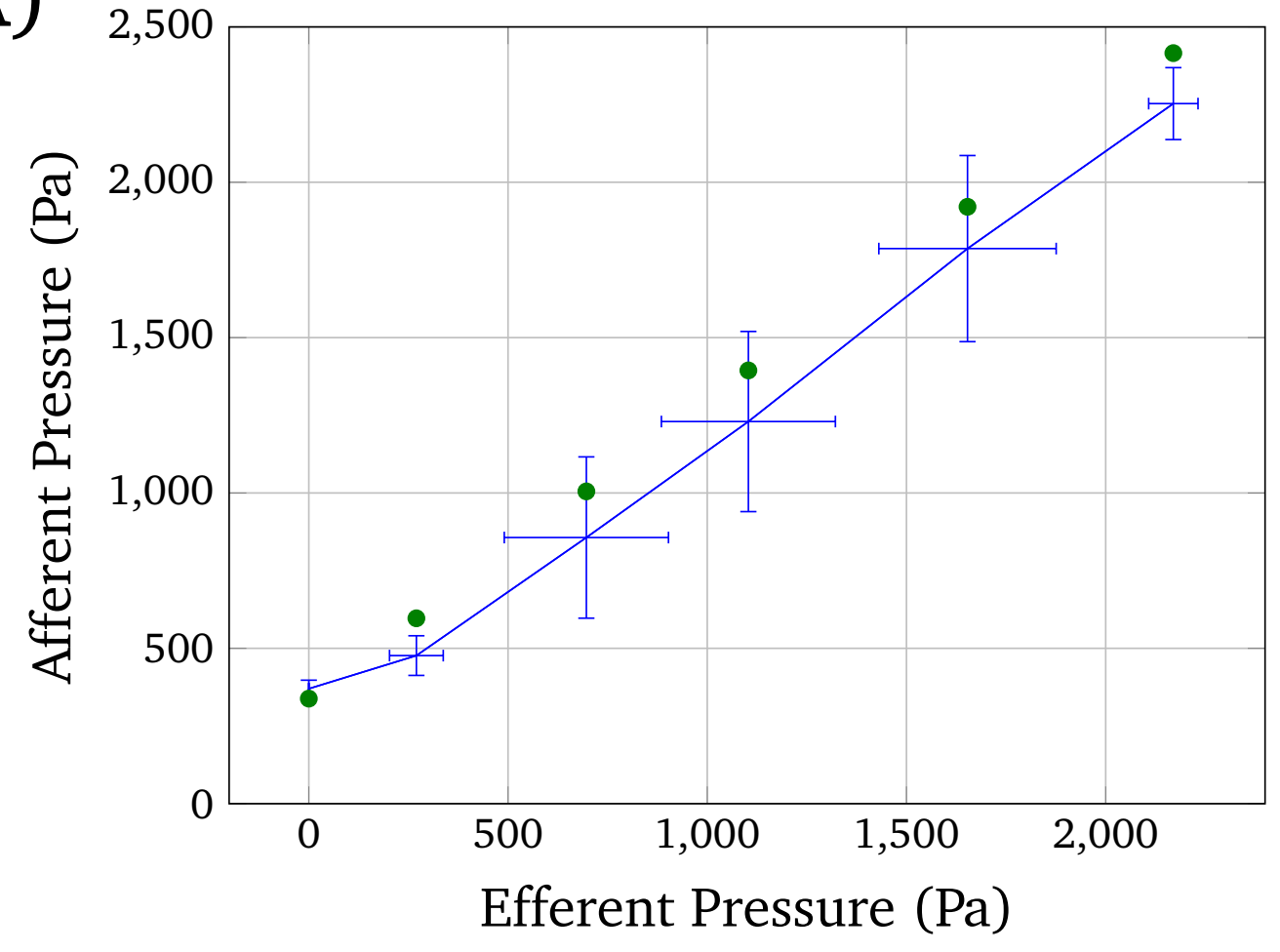

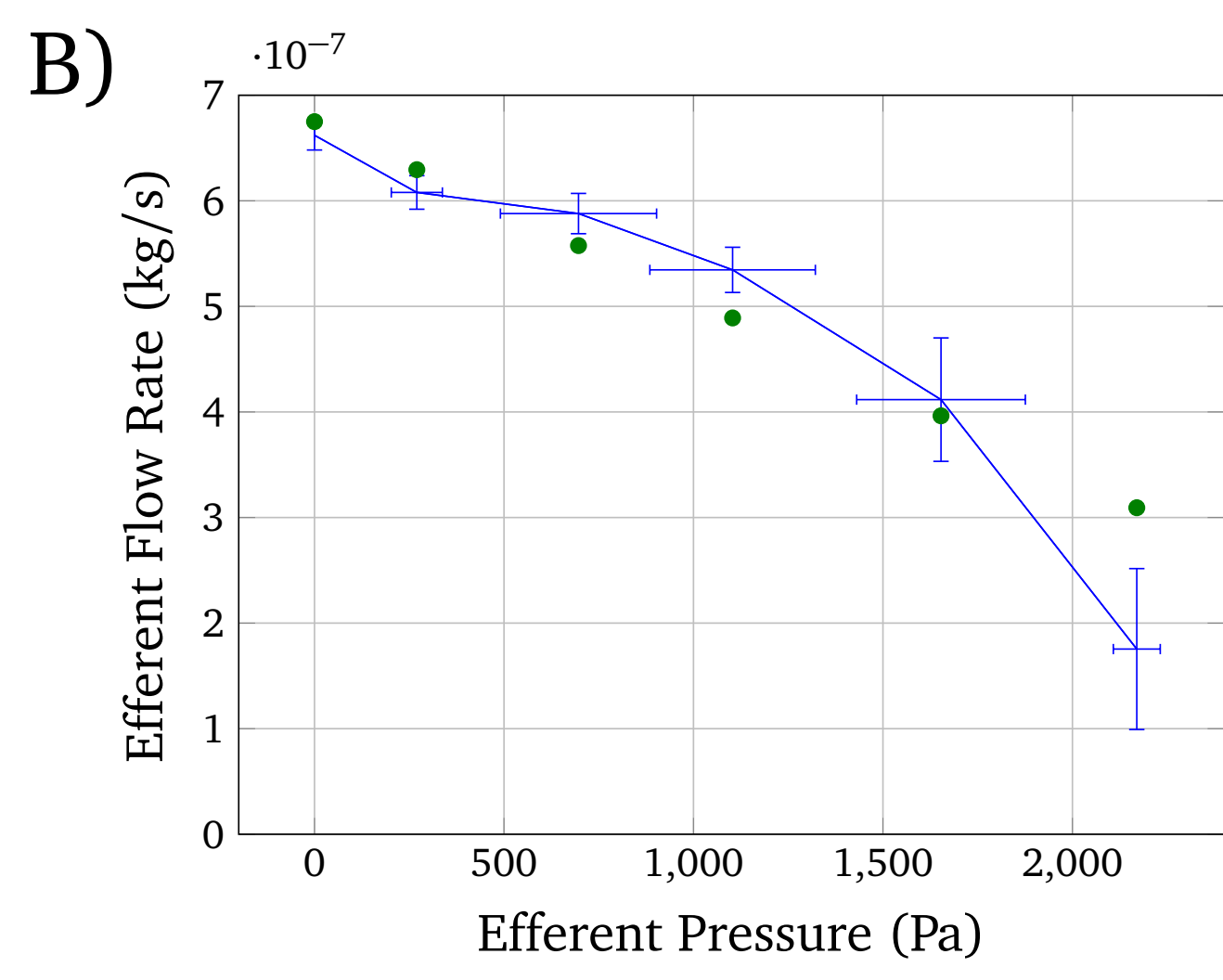


A)



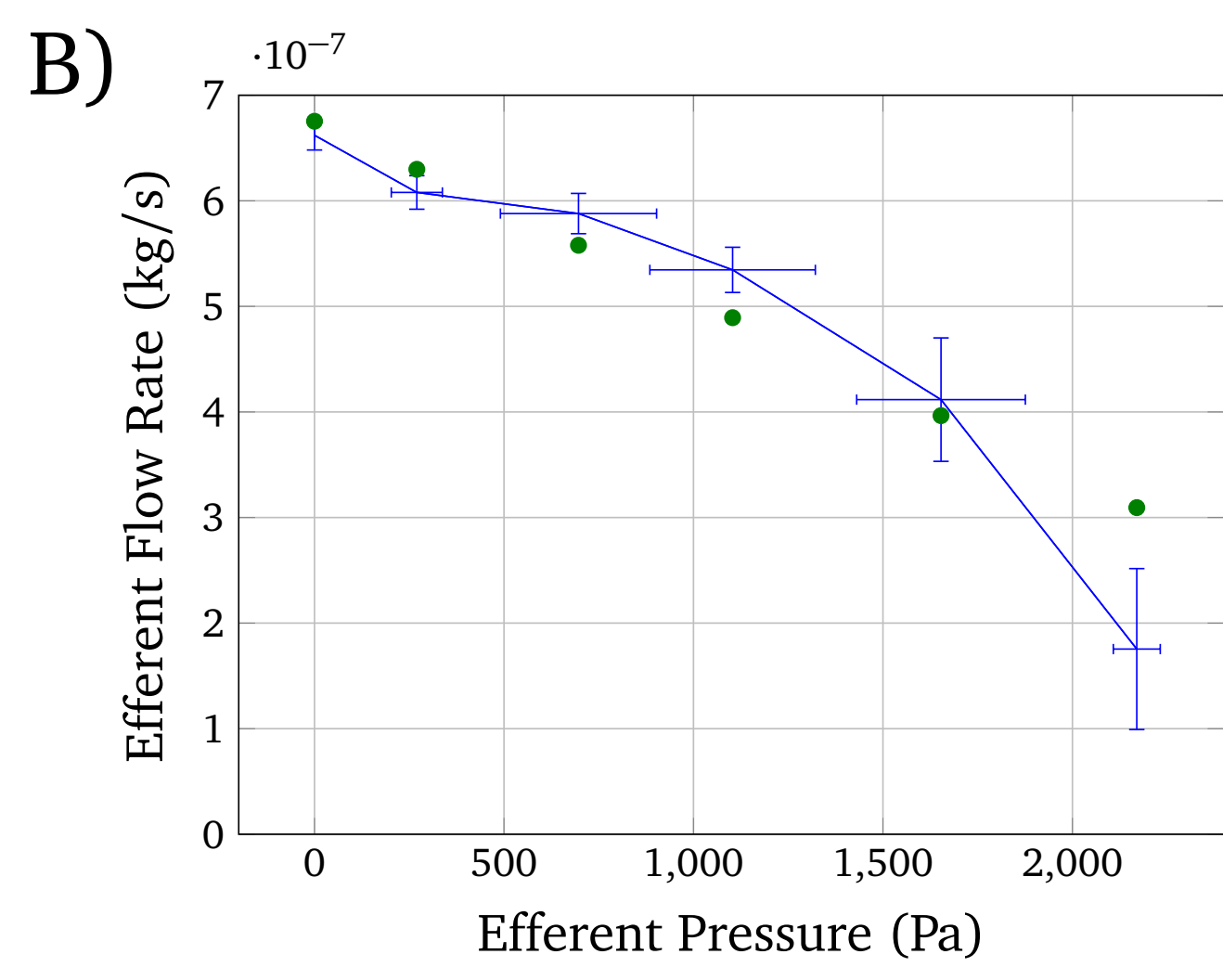



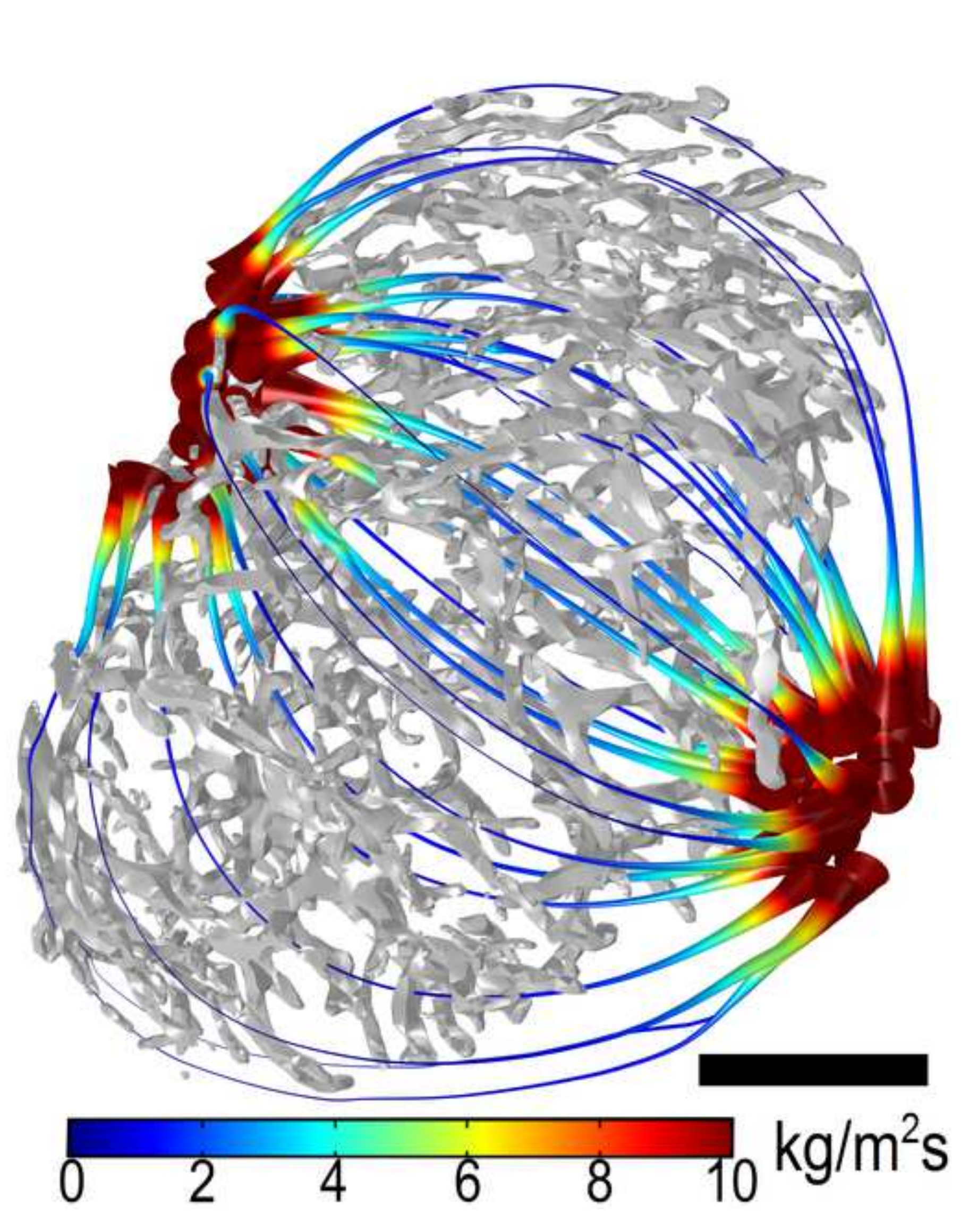

$$
\text { . }
$$
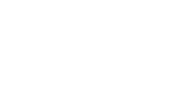

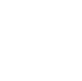



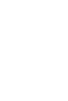
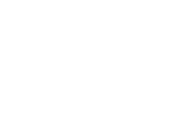

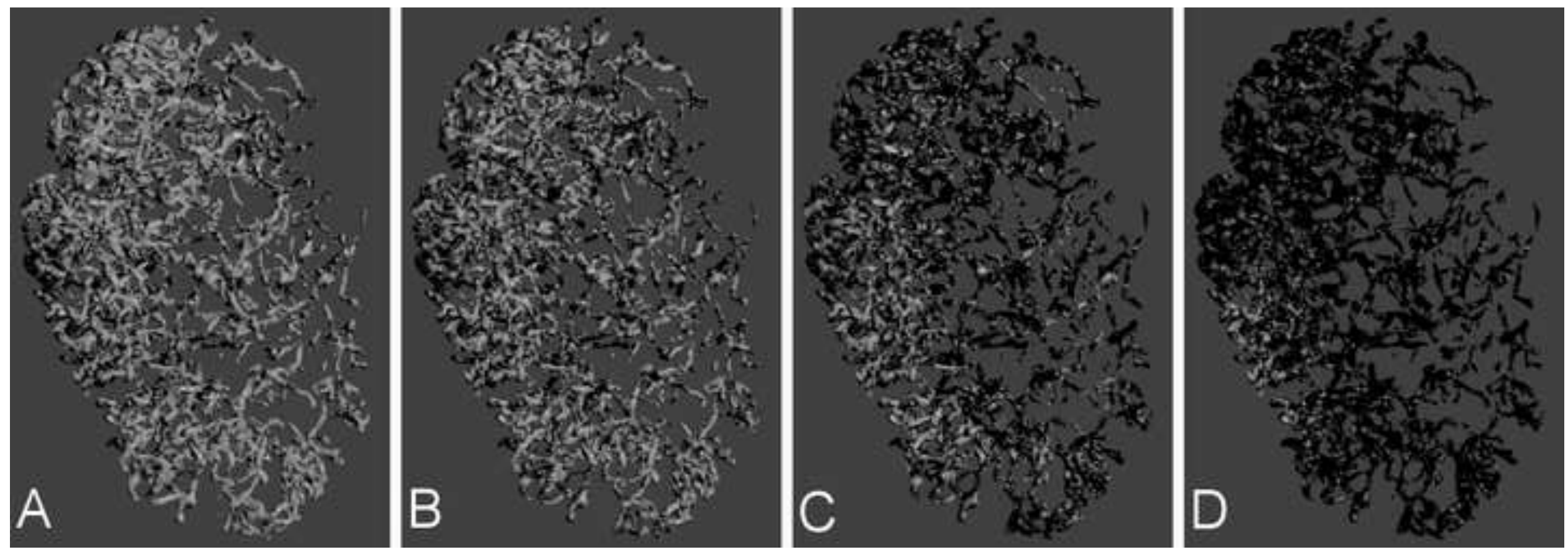


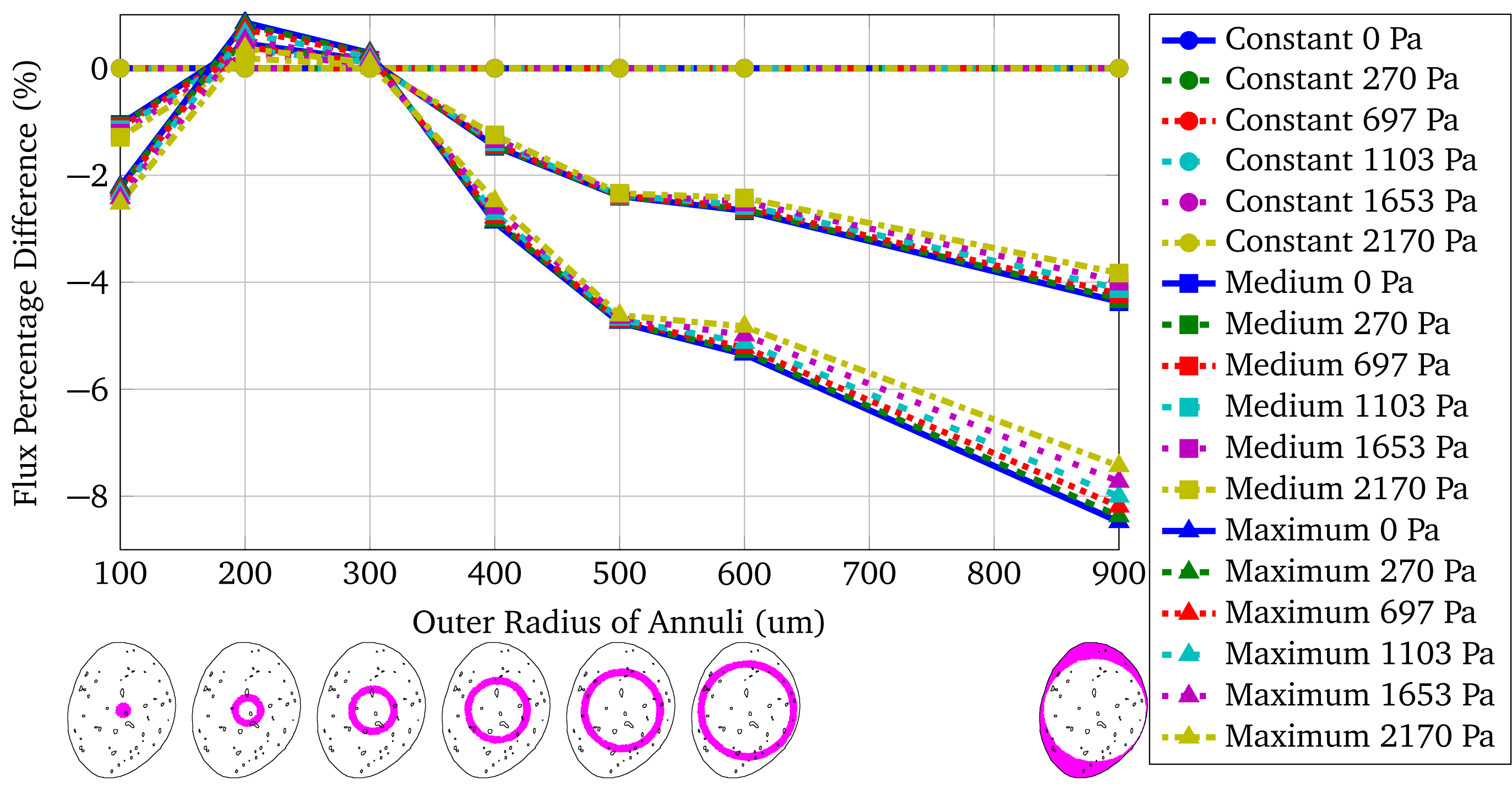




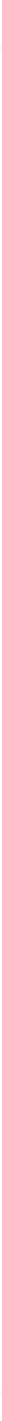


A)



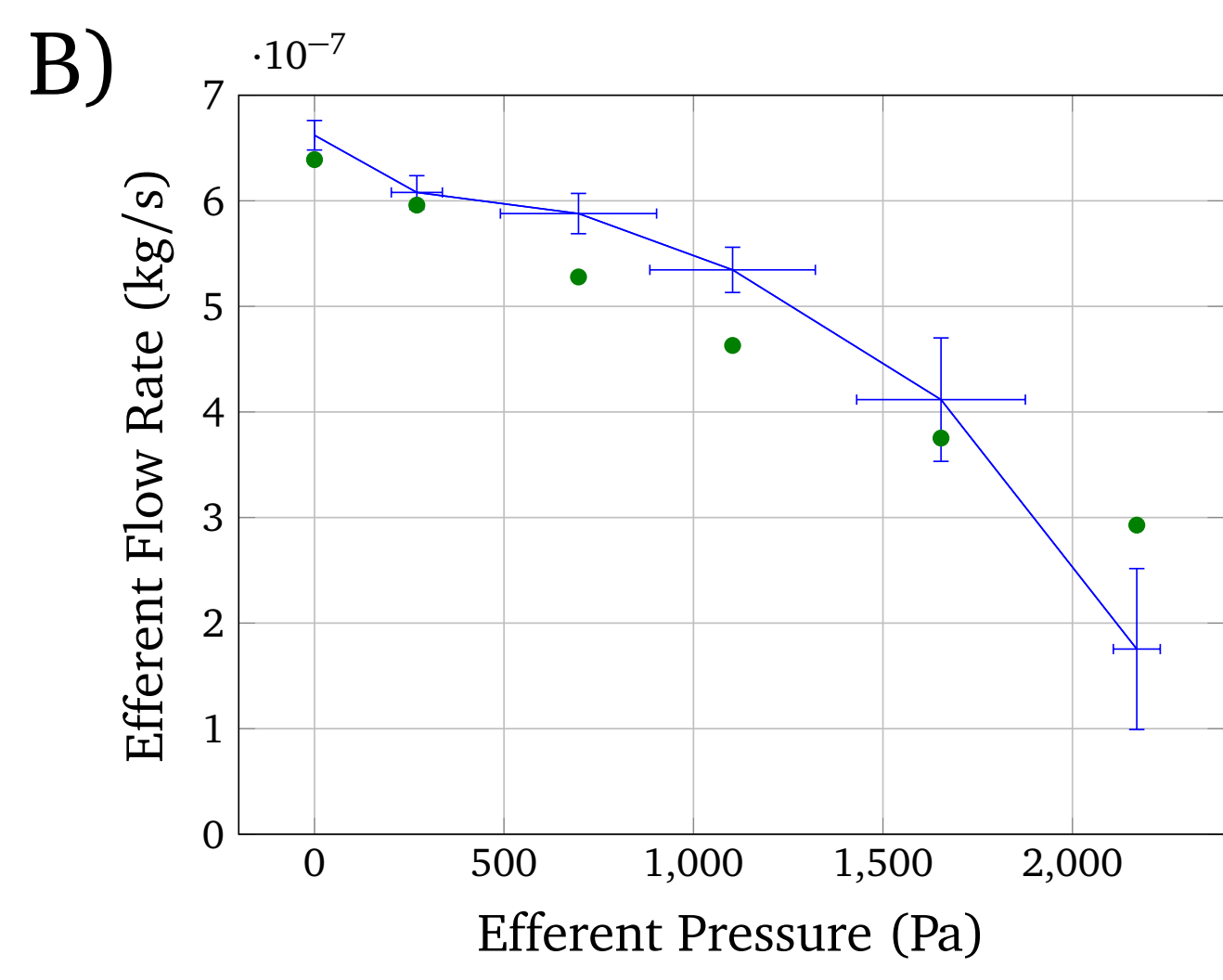

